\title{
Nonlinear Fourier transform for optical data processing and transmission: Advances and perspectives
}

\author{
Sergei K. Turitsyn ${ }^{1,2, *}$, Jaroslaw E. Prilepsky ${ }^{1}$, Son Thai LE ${ }^{3}$, Sander \\ WAHLS $^{4}$, LeOnid L. Frumin ${ }^{2,5}$, Morteza Kamalian ${ }^{1}$, And Stanislav A. \\ DEREVYANKO ${ }^{6}$
}

\author{
${ }^{1}$ Aston Institute of Photonic Technologies, Aston University, Birmingham B4 7ET, United Kingdom \\ ${ }^{2}$ Novosibirsk State University, Novosibirsk 630090, Russia \\ ${ }^{3}$ Nokia Bell Labs, Stuttgart, Germany \\ ${ }^{4}$ Delft Center for Systems and Control, Delft University of Technology, 2628 CD, Delft, The Netherlands \\ ${ }^{5}$ Institute of Automation and Electrometry, Siberian Branch, Russian Academy of Sciences, Novosibirsk 630090, Russia \\ ${ }^{6}$ Department of Electrical and Computer Engineering, Ben-Gurion University of the Negev, Beer Sheva 84105, Israel \\ *Corresponding author: s.k.turitsyn@aston.ac.uk
}

Fiber-optic communication systems are nowadays facing serious challenges due to fast growing demand on capacity from various new applications and services. It is now well recognised that nonlinear effects limit the spectral efficiency and transmission reach of modern fiber-optic communications. Nonlinearity compensation is therefore widely believed to be of paramount importance for increasing the capacity of future optical networks. Recently, there has been a steadily growing interest in the application of a powerful mathematical tool - the nonlinear Fourier transform (NFT) - in the development of fundamentally novel nonlinearity mitigation tools for fiber-optic channels. It has been recognized that, within this paradigm, the nonlinear crosstalk due to the Kerr effect is effectively absent, and fiber nonlinearity due to Kerr effect can enter as a constructive element rather than a degrading factor. The novelty and the mathematical complexity of the NFT, the versatility of the proposed system designs, and the lack of a unified vision of an optimal NFT-type communication system however constitute significant difficulties for communication researchers. In this paper, we therefore survey the existing approaches in a common framework and review the progress in this area with a focus on practical implementation aspects. First, an overview of existing key algorithms for the efficacious computation of the direct and inverse NFT is given, and the issues of accuracy and numerical complexity are elucidated. We then describe different approaches for the utilization of the NFT in practical transmission schemes. After that we discuss the differences, advantages and challenges of various recently emerged system designs employing the NFT, and the efficiency estimation available up-to-date. With many practical implementation aspects still being open, our minireview is aimed at helping researchers to assess the perspectives, understand the bottle-necks, and envision the development paths in the upcoming of NFT-based transmission technologies. (C) 2014 Optical Society of America

OCIS codes: (060.1660) Coherent communications; (060.2330) Fibre optics communications; (070.4340) Nonlinear optical signal processing; (290.3200) Inverse scattering. 


\section{INTRODUCTION}

The exponential surge in global data traffic driven by the skyrocketing proliferation of different bandwidth-hungry on-line services, like cloud computing, on-demand HD video streams, on-line business analytics, etc., brings about the escalating pressure on the speed (capacity) and quality (bit error rate) characteristics of information flows interconnecting individual network participants [1-5]. Optical fiber systems are the backbone of the global telecommunication networks. It is hard to overstate the impact that fiber communications has made on the economy, public services, society, and almost all aspects of our lives. It is also well recognized [3-12] that rapidly increasing data rates in the core fiber communication systems are quickly approaching the limits of current transmission technologies, many of which were originally developed for linear (e.g. radio) communication $[13,14]$.

Optical fiber channels are very different from wireless and other traditional linear channels. The main order effect here is the signal attenuation due to fiber loss that is compensated by optical amplifiers, e.g. the Erbium-doped amplifiers (EDFA) or distributed Raman amplification (DRA) [1]. Optical amplification adds the amplifier spontaneous emission (ASE) noise that mixes with the signal during the transmission. In general, optical noise together with dispersion and nonlinearity are the three key physical effects having major impact on signal transmission in optical fiber links. The successful implementation of the "fifth generation" of optical transmission systems, operating with coherent detection, wavelength division multiplexing (WDM), advanced multilevel modulation formats, and digital signal processing (DSP), has led to the possibility of channel rates exceeding $100 \mathrm{~Gb} / \mathrm{sec}[2,5,7]$. The key to this breakthrough is the digital mitigation of the most important linear transmission impairments, such as the chromatic and polarization mode dispersion $[1,2,30]$. After the equalization of linear effects, noise and nonlinearity become the principal factors deteriorating the performance of optical networks. Indeed, the Kerr nonlinear effect at high signal powers (and, respectively, high signal-to-noise ratio, SNR), leads to power-dependent nonlinear transmission signal distortions in the fiber channel. In this sense, the fiber nonlinearity has a detrimental effect on transmission of information and, thereby, serious world-wide efforts are aimed at the suppression or compensation of nonlinear impairments. It was stressed in [6] that, in contrast to linear channels [13], the spectral efficiency of the optical fiber WDM systems cannot be increased infinitely and starts to decay at high signal powers due to the spectral channel cross-talk imposed by fiber nonlinearity. The nonlinear fiber effects are behind the infamous "nonlinear capacity limit" problem [5, 7, 9, 10, 15].

In spite of the immense recent progress in optical communication technologies, the next step in the future systems' design has appeared to be not so straightforward [16]. Spacedivision multiplexing (SDM) is considered by many engineers as a promising direction in the evolution of optical transmission systems [17]. However, the SDM technology requires a considerable upgrade in the infrastructure. The compensation of nonlinearity-induced effects is a principal research and engineering challenge and it is likely to remain so in the future. There have been proposed a plethora of various nonlinearity compensation methods, including digital back-propagation (DBP) [18], digital [19] and optical [20, 21] phase conjugation (spectral inversion), and phase-conjugated twin waves [22], to mention just few important advances, see reviews [16, 23]).
Note, that in the most of the compensation techniques, the fiber nonlinearity is treated as an undesirable effect, and the purpose of all those methods is just to mitigate or suppress its impact.

There is, however, an alternative and not yet widely popular view-point: since fiber channels are inherently nonlinear, rather than treating nonlinearity as a completely destructive feature, it can be considered as an essential element in the design of fiber communication systems. There is a growing evidence of the necessity of a novel paradigm and radically new approaches to coding, transmission, and processing of information, that would take into account the nonlinear properties of the optical fiber. In this work we describe one of such recently resurrected approaches-the NFT. The NFT-based transmission method belongs to a conceptually different bevy of techniques compared to those mentioned above [24]: Here the nonlinearity enters as an undetachable element of the processing and transmission, defining the features of the system architecture and its characteristics. The application of such paradigm-shifting nonlinear methods means that some common "linear" methodology may need to be reconsidered or appended with a new meaning. For instance, in addition to the usual notions of frequency, spectral power, and bandwidth, one has to work with their nonlinear analogues that can be drastically non-conventional, but can serve as new well-defined and adjustable characteristics of the optical signal in nonlinear systems. It will be convenient further to distinguish between signal characteristics in the standard frequency domain and those in the so-called nonlinear spectral domain. Note also that for the sake of clarity within this review we address only the single-mode and single-polarization fiber transmission model, leaving aside the polarization degree of freedom and specific peculiarities of multimode systems [5].

We would like to stress that the beauty of the mathematical theory presented here is inevitably spoiled by the limits of applicability of the master model - the integrable nonlinear Schrödinger equation (NLSE) - for the description of signal transmission in fiber links. The application of the NFT methods is limited by deviations of the optical signal dynamics from the NLSE channel model. Apart from the deviations due to periodic variations of signal power caused by alternation of loss and gain in practical systems (in that case the NLSE emanates as a leading approximation within the path-averaged model), various other effects contribute to perturbations that are not accounted for by the pure NLSE, e.g. higher-order dispersion [2527], polarization effects [26, 28-30], the Raman effect [26, 27, 31], the acoustic effects (electrostriction) [32], all limit the validity of this channel model. Consideration of the impact of these effects is beyond the scope of this survey which is focused on the NFT techniques for the NLSE-based channel.

To assist reading of the paper, Section 1 of the Supplementary material contains the list of acronyms used on our review.

\section{PRINCIPLES OF INTEGRABILITY AND NFT}

In physics and, notably, in photonics, many important phenomena and the evolution of underlying systems can be modelled by the NLSE [1, 26, 27,33-36]. In particular, the NLSE is a principal master model governing the evolution of the slow-varying optical field envelope $q(z, t)$ ( $z$ will further play the role of the distance along the fiber while $t$ is the time variable) along a single-mode fiber,

$$
i \frac{\partial q}{\partial z} \pm \frac{1}{2} \frac{\partial^{2} q}{\partial t^{2}}+|q|^{2} q=0
$$


Note that this is NLSE in its normalized form. Here and in what follows, the upper sign in Eq. (1) ("+") corresponds to anomalous fiber dispersion, while the lower one ("-") refers to the normal dispersion case. Formally, the NLSE (1) describes the evolution of light in a lossless optical fiber under the effects of dispersion and Kerr nonlinearity. Albeit all real fibers, certainly, have losses, this model appears as a result of averaging over periodic gain and loss variation, leading to effectively conservative signal evolution [26, 27, 33, 34]. Close to ideal compensation of losses along optical fiber is possible in specific schemes of the so-called ultra-long fiber lasers DRA [37, 38]. Such a quasi-lossless transmission was demonstrated experimentally in $[38,39]$.

A possibility to approximate signal evolution in practical fiber channels by the NLSE gives a remarkable opportunity to apply advanced mathematical techniques, developed in 1970s, to optical communications. It was first shown in the seminal work by Zakharov and Shabat [40], that Eq. (1) belongs to the class of the so-called integrable nonlinear systems. The mathematical method, widely known in the physical and mathematical communities as the inverse scattering transform (IST), can be applied to find the solution of integrable nonlinear equations. In this context one can think of integrability as an elegant transform of the original nonlinear system into the so-called actionangle variables corresponding to a set of uncoupled trivial evolutionary equations. Mathematically, this can be treated as an effective linearization of the nonlinear evolution. There exists a vast amount of literature where the integrability notion is elucidated in great detail, see e.g. [27, 35, 36, 40-44]. The integrability itself implies a lot of consequences in both mathematical and physical contexts. For example, the NLSE (1) possesses an infinite number of conserved quantities: While the conservation of power, momentum and Hamiltonian for Eq. (1) is relatively obvious, the rest of the conserved quantities are nontrivial. As we will show below, a lot of fruitful ideas based on the conserved quantities have been successfully implemented.

In a nutshell, NFTs can be used to solve initial value problems for a special class of nonlinear evolutionary equations. In fiber optics, where the signal evolution occurs along the fiber, the initial conditions correspond to the time domain waveform at the transmitter. Similar to conventional Fourier transform (FT), initial conditions of the integrable nonlinear equations (such as, e.g. NLSE, or a Manakov system [47], corresponding to the integrable two-component NLSE generalization) can be decomposed into (nonlinear) spectral data. For the NLSE this is done through the solution of a linear scattering problem known as the Zakharov-Shabat problem (ZSP) [40,41]. Any solution of the NLSE can be presented as the evolution of nonlinear spectral data that evolve effectively in a linear manner. The inverse transform, namely the recovery of the space-time domain field distribution from the known nonlinear modes, is clasically implemented through solution of the Gelfand-Levitan-Marchenko equation (GLME). Altogether, this means that such a spectral transform can be interpreted as the direct nonlinear analog of the FT [35, 40, 41]: similarly to the FT transforming the dispersion of a linear propagation to a phase rotation in frequency space, the NFT recasts both the nonlinearity and dispersion of the NLSE into a simple decoupled evolution of nonlinear spectral data inside the NFT domain (see Fig. 1); the latter plays the role of Fourier spectrum for nonlinear problems.

By performing the NFT of a given profile $q(z, t)$, we segregate two distinct components: the dispersive nonlinear radiation and the non-dispersive solitons, although either of these two can be absent for specific profiles. For normal dispersion, the inputs localised in time cannot nucleate solitons. For the dispersive part of the nonlinear spectrum, the NLSE evolution produces exactly the linear phase rotation of spectral components as we have for linear systems. For the anomalous dispersion, the solitons, associated with the complex "nonlinear frequencies" (eigenvalues), in addition to the rotation of soliton phases can involve either the motion as a whole or a more nontrivial beating dynamics of bound states-the so-called multi-soliton breathers [27,33], although inside the NFT domain the solitonic degrees of freedom remain decoupled. Note that NFT methods are much richer, more flexible and versatile with respect to the system design and performance compared to just soliton-based techniques, studied previously in many details [26, 27, 33]. In the NFT methods dealing with the discrete part of nonlinear spectrum (solitonic eigenvalues), the information carriers are not the fundamental solitons themselves but the NFT parameters (nonlinear spectral data) attributed to a multisolution pulse. In this sense, the traditional soliton-based transmission emerges as the simplest (and not necessarily optimal) subclass of the NFT methods. The NFT communications are, to some extent, the extension of not only the soliton-based approach but also of the coherent communication idea itself: While for the latter both signal's amplitude and phase are used for modulation, the NFT approach goes further and employs the nonlinear characteristics of the signal.

Thus, by using the NFT all effects caused by the fiber Kerr nonlinearity can be described as a trivial change of the phase in the nonlinear spectrum. This paves the way to fundamentally novel nonlinear techniques for compensation of the effects of chromatic dispersion and fiber nonlinearity. In 1993 Hasegawa and Nyu [42] (see also Chapter 4 of [27]) proposed the truly innovative idea of eigenvalue communications based on the exploitation of discrete eigenvalues (corresponding to solitons) emerging in the NFT signal decomposition to encode and transmit information [40-42]. This approach potentially solves the problem of nonlinear cross-talk that is one of the major challenges in optical WDM systems. The concept of Hasegawa and Nyu has been recently resurrected with various modifications and further extensions [24, 44-46, 48-56, 58, 60-77], including the new direction employing the modulation of continuous nonlinear spectrum [51-56, 58, 60, 61, 77], first experiments using transmission and processing of discrete eigenvalues [62-65], NFT-based DBP [50, 73] and, most recently, the polarization division multiplexing with the NFT [72]. The transition from the space-time domain into the nonlinear spectral domain and back is achieved by performing the NFT operations. Generally, there exists the straightforward interrelation not only in the ideology of FT and NFT methods but also between the linear and NFT spectra [35]: In the low power limit one can prove the asymptotic equivalence of the linear FT and NFT [41]. However, in spite of the similarity, the explicit form of the NFT operations is much more mathematically involved as compared to the simple profile convolution with exponentials for the usual FT. Thus, the complexity of the NFT operations and the "change of notions" often bring about the difficulties for the communication engineers. The purpose of our survey is to demonstrate without deep mathematical details how the NFT method and various integrability features can be employed for the the sake of efficient optical transmission, also summing up the existing numerical tools that can be employed for the computation of the NFT.

This paper is organized as follows. First, in Section 3 we describe the the NLSE model for realistic optical fiber systems. 
In Section 4 we introduce minimally required notations for the NFT operations, including the periodic NFT variant. Then, in Section 5 we overview the existing numerical methods for the calculation of the direct and inverse NFT operations. Section 5 $C$ is focused on fast NFT algorithms. Then, in Section 6 we directly address different NFT-based transmission methods, also presenting some new results and generalizations. After that, in Section 7 we overview recent results with regard to the efficiency of NFT-based optical transmission mehods. The paper ends with the Conclusion, where we also outline some NFT perspectives and further development directions.

\section{GENERALIZED NLSE MODEL OF OPTICAL FIBER}

The principal master model for the electrical field $q(z, t)$ evolution inside a single-mode optical fiber with the account of amplification can be written as generalized NLSE (GNLSE) $[1,26,27]$ :

$$
i \frac{\partial q}{\partial z}-\frac{\beta_{2}}{2} \frac{\partial^{2} q}{\partial t^{2}}+\gamma|q|^{2} q=i g(z) q+\eta(z, t),
$$

with $z$ being a distance (in $\mathrm{km}$ ) along the fiber, $t$ is time (in ps) in the frame co-moving with the velocity of the envelope. The parameter $\beta_{2}$ (in $\mathrm{ps}^{2} / \mathrm{km}$ ) is the characteristic of chromatic dispersion that is negative for the anomalous dispersion (the most important practical case) or $\beta_{2}>0$ for the normal dispersion $\left(\left|\beta_{2}\right|\right.$ can vary from $5 \mathrm{ps}^{2} / \mathrm{km}$ to $\left.60 \mathrm{ps}^{2} / \mathrm{km}\right)$; further, for a standard single mode-fiber we assume $\beta_{2}=-22 \mathrm{ps}^{2} / \mathrm{km}$. $\gamma$ is the nonlinear Kerr coefficient, typically $\gamma=1.27 \mathrm{~W}^{-1} \mathrm{~km}^{-1}$. The function $g(z)$ characterizes the gain-loss profile of a particular amplification scheme. For the quasi-lossless DRA scheme, the function $g(z) \equiv 0[38,39]$, resulting in the lossless NLSE perturbed by the additive white Gaussian noise (AWGN) term $\eta(z, t)$ (having zero mean). The latter is completely characterized by ASE spectral power density $D$ :

$$
\mathbb{E}\left[\eta(z, t) \bar{\eta}\left(z^{\prime}, t^{\prime}\right)\right]=2 D \delta\left(t-t^{\prime}\right) \delta\left(z-z^{\prime}\right),
$$

where the overbar means the complex conjugate, $\mathbb{E}(\cdot)$ is the expectation value and $\delta(\cdot)$ is the Dirac delta-function. In the case of ideal DRA we have: $2 D=h v_{0} K_{T} \alpha$, where $\alpha$ is the fiber loss coefficient, typically $\alpha \approx 0.2 \mathrm{~dB} / \mathrm{km}$ at the carrying wavelength $\lambda_{0}=1.55 \mu \mathrm{m}, K_{T}$ is the temperature-dependent factor (related to the phonon-occupancy factor) that characterizes the Raman pump providing the distributed gain, $K_{T}$ is typically in the range from 1.1 to $1.2 ; v_{0}$ is the carrying frequency of the signal corresponding to $\lambda_{0}: v_{0}=193.55 \mathrm{THz}$. Taking these typical values of parameters, one estimates the order of characteristic noise intensity per complex signal component (polarization), per unit of propagation length and per unit of bandwidth, to be $D \sim 10^{-21} \mathrm{~J} / \mathrm{km}$; for $K_{T}=1.13$ we have: $D \approx 3.3 \cdot 10^{-21}$ $\mathrm{J} / \mathrm{km}$. Such an idealized form of optical channel (the lossless integrable NLSE (1) weakly perturbed by the AWGN) suits NFT applications [52] best as it is close to the integrable NLSE (1).

However, the NFT method can still be successfully applied to the EDFA (lumped) [53] or non-ideal DRA [54, 55] cases. For the EDFA we have $g(z)=-\alpha$ in between point-action (lumped) amplifiers, but the signal is boosted to the initial power level after each span of length $Z_{a}$. For the DRA scheme we have a more complicated non-flat profile of $g(z)$, e.g. that corresponding to the open-cavity random distributed feedback laser-based amplification as it provides the best performance among various other Raman amplification schemes [78], where the gain profile recurs periodically after each span of the length $Z_{a}$. Now, by using the path-averaged approach [26, 27, 34, 53-55] one can introduce the new field variable as $\tilde{q}(z, t)=q(z, t) G^{1 / 2}(z)$, where $G(z)=\exp \left[2 \int_{0}^{Z_{a}} g(z) d z\right]$, and this substitution recasts Eq. (2) into the lossless NLSE for $\tilde{q}(z, t)$ with the $z$-dependent factor $G(z)$ near the nonlinear term. In the leading order with respect to $Z_{a} / Z_{d}$, with $Z_{d}$ being the dispersion length, $\left[Z_{d}=\right.$ $\left(W^{2}\left|\beta_{2}\right|\right)^{-1}$, where $W$ is the signal's bandwidth], the distancedependent nonlinearity coefficient can be approximated with the averaged value $\tilde{\gamma}=\gamma Z_{a}^{-1} \int_{0}^{Z_{a}} G(z) d z$, such that we arrive at the lossless path-averaged (LPA) NLSE written for $\tilde{q}(z, t)$ with constant coefficients with $\tilde{\gamma}$ in place of original $\gamma$ from (2); for the EDFA system $\tilde{\gamma}=\gamma\left(G_{a}-1\right) / \ln G_{a}$ with $G_{a}=\exp \left(-2 \alpha Z_{a}\right)$. In general, the applicability limits of the LPA NLSE model depend on the signal power, bandwidth and transmission distance. The accuracy of the LPA NLSE for optical links with EDFA was investigated in [53] for a link distance of $2000 \mathrm{~km}$, signal powers up to $8 \mathrm{dBm}$ and bandwidths up to $80 \mathrm{GHz}$. It was found that the LPA NLSE model can be applied with a normalized mean square error below $-20 \mathrm{dBm}$ when the signal power is below $3 \mathrm{dBm}$, almost independently of the signal bandwidth. The LPA model was found to work under more relaxed requirements with non-ideal RDA [55] as this amplification scheme provides a lower gain variation along the link, depending on the specific RDA scheme. The applicability limits of this model for the EDFA case with regards to NFT applications were presented in [53], and for the RDA scheme - in [55]. The noise term is assumed to possess the same properties as we have for the ideal RDA case, i.e. it is the circular AWGN with only a different expression for the intensity $\tilde{D} \sim 10^{-21}$ $\mathrm{J} / \mathrm{km}$. For the EDFA system $2 \tilde{D}=n_{\mathrm{sp}} h v_{0}\left(G_{a}^{-1}-1\right) / Z_{a}$, where $n_{\mathrm{sp}} \approx 1$ is the spontaneous emission factor [33].

Having recast our GNLSE (2) to the approximate LPA NLSE form with the distance-independent coefficients or using the ideal RDA model, we introduce the normalizations,

$$
t / T_{S} \rightarrow t, \quad z / Z_{s} \rightarrow z, \quad q / \sqrt{P_{0}} \rightarrow q,
$$

with $P_{0}=\left(\gamma Z_{s}\right)^{-1}$ (or the same with the $\tilde{\gamma}$ for EDFA or nonideal DRA and resulting LPA NLSE), $Z_{s}=T_{s}^{2} /\left|\beta_{2}\right|$, and we finally have the standard NLSE model, Eq. (1), but with the AWGN term in the r.h.s. In Eq. (4) either of three parameters, $T_{s}, Z_{s}$, or $P_{0}$, can be taken for the normalization, but then the remaining two have to be properly adjusted: $T_{S}$ can be, e.g., the extent of our symbol, or setting it to be the reciprocal bandwidths, $T_{S}=W^{-1}$, our normalized distance unit becomes the dispersive lengths mentioned above; in soliton-related problems $T_{S}$ is often set as an individual soliton full width at half maximum (FWHM). The noise intensity has to be normalized in accordance to (4): $D Z_{\mathcal{S}}\left(P_{0} T_{S}\right)^{-1} \rightarrow D$. We also omit tildes in $\tilde{q}, \tilde{\gamma}$, $\tilde{D}$ further, assuming that Eq. (1) refers to a simplified description pertaining to a particular amplification scheme. Of course, the results for the NFT application for non-ideal DRA or EDFA schemes are expected to show a slightly worse performance as compared to the ideal DRA [53-55], thought the higher order corrections with respect to $Z_{a} / Z_{d}$ may also be taken into account by using, e.g., the guiding center approximation [27].

\section{EXPLICIT FORM OF NFT OPERATIONS}

In this section, the direct (forward) and inverse NFT (INFT) are introduced. The NFT considers the signal $q(z, t)$ at a fixed location $z=z_{0}$ and returns the corresponding NFT spectrum. The INFT reverses this process, i.e., given a NFT spectrum it returns 
the corresponding signal $q\left(z_{0}, t\right)$. Since only the main features can be outlined here, the reader is referred to [27, 35, 40-46] for further details. The section ends with some properties of the periodic NFT.

\section{A. Direct NFT}

The direct NFT is computed from specific (auxiliary) solutions $v_{1,2}(t, \zeta)=v_{1,2}\left(t, \zeta ; z_{0}\right)$ to the ZSP [40]

$$
\frac{d v_{1}}{d t}=q\left(z_{0}, t\right) v_{2}-i \zeta v_{1}, \quad \frac{d v_{2}}{d t}=\mp \bar{q}\left(z_{0}, t\right) v_{1}+i \zeta v_{2},
$$

for different values of the complex parameter $\zeta=\xi+i \eta$, which will play the role of a nonlinear analog of frequency. The signal $q(z, t)$ acts as a potential. The upper and lower signs correspond to the anomalous and normal dispersion according to Eq. (1). Under the assumption that $q\left(z_{0}, t\right)$ decays at least exponentially for $t \rightarrow \pm \infty$, specific solutions (the so-called Jost functions) $\phi_{1,2}(t, \zeta)$ and $\psi_{1,2}(t, \zeta)$ to the ZSP can be obtained from the boundary conditions:

$$
\begin{gathered}
\phi_{1}(t, \zeta)=e^{-i \zeta t}+o(1), \quad \phi_{2}(t, \zeta)=o(1) \text { for } t \rightarrow-\infty, \\
\psi_{1}(t, \zeta)=o(1), \quad \psi_{2}(t, \zeta)=e^{i \zeta t}+o(1) \text { for } t \rightarrow+\infty .
\end{gathered}
$$

In practical realization of the transmission schemes the pulse $q(t)$ is truncated and we operate in the so-called burst mode [52], see Fig. 2. The above pairs of functions solve the ZSP and all these different solutions are linearly dependent:

$$
\begin{gathered}
{\left[\begin{array}{ll}
\phi_{1} & \phi_{2}
\end{array}\right]=a(\zeta)\left[\begin{array}{ll}
\tilde{\psi}_{1} & \tilde{\psi}_{2}
\end{array}\right]+b(\zeta)\left[\begin{array}{ll}
\psi_{1} & \psi_{2}
\end{array}\right]} \\
{\left[\begin{array}{ll}
\tilde{\phi}_{1} & \tilde{\phi}_{2}
\end{array}\right]=-\tilde{a}(\zeta)\left[\begin{array}{ll}
\psi_{1} & \psi_{2}
\end{array}\right]+\tilde{b}(\zeta)\left[\begin{array}{ll}
\tilde{\psi}_{1} & \tilde{\psi}_{2}
\end{array}\right] .}
\end{gathered}
$$

The functions $a(\zeta)$ and $b(\zeta)$ are known as the Jost scattering coefficients. They serve as the basis on which the NFT spectrum is defined. Due to the boundary conditions, we have

$$
a(\zeta)=\lim _{t \rightarrow \infty} \phi_{1}(t, \zeta) e^{i \zeta t}, \quad b(\zeta)=\lim _{t \rightarrow \infty} \phi_{2}(t, \zeta) e^{-i \zeta t}
$$

Another important property of the Jost scattering coefficients is that they satisfy $|a(\xi)|^{2} \pm|b(\xi)|^{2}=1$ for all real $\xi$, where the upper and lower signs refer to those in Eqs. (1) and (5). The NFT spectrum of the signal $q\left(z_{0}, t\right)$ consists of two parts. The first part is given either by the left or right reflection coefficient $(\mathrm{RC})$, respectively:

$$
l(\xi)=\bar{b}(\xi) / a(\xi), \quad r(\xi)=b(\xi) / a(\xi), \quad \xi \in \mathbb{R} .
$$

The second part of the NFT spectrum consists of the discrete eigenvalues $\zeta_{n}=\xi_{n}+i \eta_{n}$, which are the eigenvalues of the ZSP with a positive imaginary part $\eta>0$, and their associated left or right norming constants (also ofter referred to as spectral amplitudes), which are defined by the residue of $l(\zeta)$ (or $r(\zeta)$ ) at the point $\zeta_{n}$ :

$$
l_{n}=\left[b\left(\zeta_{n}\right) a^{\prime}\left(\zeta_{n}\right)\right]^{-1}, \quad r_{n}=b\left(\zeta_{n}\right) / a^{\prime}\left(\zeta_{n}\right),
$$

where the prime designates the derivative with respect to $\zeta$. We therefore have four real parameters defining each solitary degree of freedom. The complete (left or right) NFT spectrum of the signal $q\left(z_{0}, t\right)$ is given by

$$
\Sigma_{l}=\left\{l(\xi),\left[\zeta_{n}, l_{n}\right]_{n=1}^{N}\right\}, \quad \Sigma_{r}=\left\{r(\xi),\left[\zeta_{n}, r_{n}\right]_{n=1}^{N}\right\},
$$

where $N$ is the total number of solitons in the signal; an exemplary NF spectrum is shown in Fig. 1. The NF spectrum

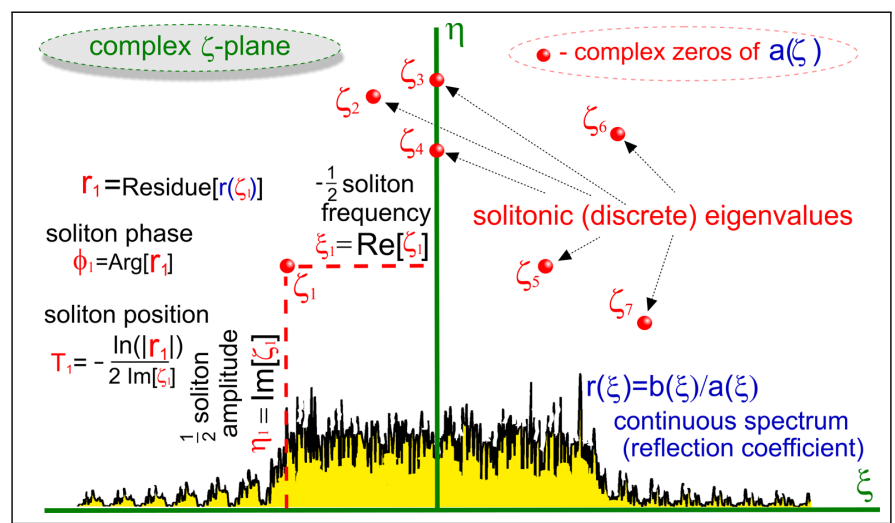

Fig. 1. An exemplary NF spectrum (anomalous dispersion case), containing solitons (discrete eigenvalues) and continuous nonlinear spectrum (depicted on the real axis $\xi$ ).

characterizes the signal $q\left(z_{0}, t\right)$ completely and can be used to recover the corresponding time-domain signal given that it vanishes sufficiently fast for $|t| \rightarrow \pm \infty$. Note that in the normal dispersion case, the signal cannot have solitonic components and either $l(\xi)$ or $r(\xi)$ are sufficient to uniquely recover the corresponding profile $q\left(z_{0}, t\right)$. The $z$-dependence of the NF spectrum, $\Sigma_{l, r}(z)$, is given by the following expressions. The eigenvalues $\zeta_{n}$ are independent on $z$. For the remaining quantities, we have:

$$
\begin{aligned}
& l(\xi, z)=l\left(\xi, z_{0}\right) e^{-2 i \zeta^{2}\left(z-z_{0}\right)}, \quad l_{n}(\xi, z)=l_{n}\left(\xi, z_{0}\right) e^{-2 i \zeta_{n}^{2}\left(z-z_{0}\right)}, \\
& r(\xi, z)=r\left(\xi, z_{0}\right) e^{2 i \xi^{2}\left(z-z_{0}\right)}, \quad r_{n}(\xi, z)=r_{n}\left(\xi, z_{0}\right) e^{2 i \zeta_{n}^{2}\left(z-z_{0}\right)} .
\end{aligned}
$$

Finally, we remark that the solitons disappear and the NFT reduces to conventional FT when the signal power becomes small. Any rescaled signal $q_{\epsilon}(t)=\epsilon q(t)$ satisfies [35, 41]

$$
\epsilon^{-1} \bar{r}_{\epsilon}(\xi), \epsilon^{-1} l_{\epsilon}(\xi) \rightarrow-\left.q(\omega)\right|_{\omega=-2 \xi} \quad \text { when } \epsilon \rightarrow 0,
$$

where $q(\omega)=\int_{-\infty}^{\infty} q(t) e^{-i \omega t} d t$. Also note that, in optics, the ZSP (5) also appears widely in the field of Bragg grating synthesis [1,79-81], where the functions $v_{1,2}$ play the role of slowly varying coupled mode amplitudes: The anomalous dispersion [the upper sign in Eqs. (5), (16)] corresponds to the coupling of co-propagating waves while the normal dispersion (the lower sign) refers to counter-propagating modes.

\section{B. INFT operation (left set of scattering data)}

The inverse NFT (INFT) maps the scattering data $\Sigma_{l, r}$ onto the field $q(t)$ : This is classically achieved via the GLME for the unknown functions $K_{1,2}\left(t, t^{\prime}\right)$ [27, 35, 40, 41, 44]. The GLME, written in terms of the left scattering data, reads

$$
\begin{gathered}
\bar{K}_{1}\left(\tau, \tau^{\prime}\right)+\int_{-\infty}^{\tau} d y L\left(\tau^{\prime}+y\right) K_{2}(\tau, y)=0, \\
\mp \bar{K}_{2}\left(\tau, \tau^{\prime}\right)+L\left(\tau+\tau^{\prime}\right)+\int_{-\infty}^{\tau} d y L\left(\tau^{\prime}+y\right) K_{1}(\tau, y)=0,
\end{gathered}
$$

for $\tau>\tau^{\prime}$, where the upper and lower signs correspond to upper and lower ones in Eqs. (1), (5). In the realistic applications, where the operations are performed on a finite interval of $\tau$, say $0<\tau<T$, we have a finite region for the change 
of $\tau^{\prime}, \tau^{\prime}<|\tau|$. For the anomalous dispersion [the sign "-" in Eqs. (16)] the quantity $L(\tau)$ can contain contributions from both solitonic (discrete) and radiation (continuous) spectrum parts, $L(\tau)=L_{\text {sol }}(\tau)+L_{\text {rad }}(\tau)$, where

$$
L_{\mathrm{sol}}(\tau)=-i \sum_{n} l_{n} e^{-i \zeta_{n} \tau}, \quad L_{\mathrm{rad}}(\tau)=\frac{1}{2 \pi} \int_{-\infty}^{\infty} d \xi l(\xi) e^{-i \xi \tau}
$$

and we have assumed that all discrete eigenvalues have a multiplicity one. The "nonlinear time" variable $\tau$ is thus Fourier conjugated to the "nonlinear frequency" $\xi$, so that one can start not from the $\xi$-domain but immediately from the functions given by (17) in the $\tau$-domain. In this paper we have chosen to work with the left reflection coefficient, $l$, corresponding to the GLME inversion around $-\infty$. The reason for such choice is that (as we shall see below) this is a common convention in the fiber Bragg grating reconstruction problems from which we borrow most of our INFT numerical algorithms. Having solved the GLME (16) for $K_{1,2}\left(\tau, \tau^{\prime}\right)$, the sought solution in the space-time domain is recovered as $q(t)=-2 \bar{K}_{2}(t, t)$. For the soliton-free case we have $L_{\text {sol }}(\tau)=0$, and the only quantity participating in (16) is the FT of $\operatorname{RC} l(\xi): L(\tau) \equiv L_{\mathrm{rad}}(\tau)$. When one is interested in the solution $q\left(z_{0}, t\right)$ at some distance $z=z_{0}$, the quantity $l(\xi)$ in (17) is replaced with $l\left(z_{0}, \xi\right)$. The resulting solution of the GLME (16) becomes a function of $z_{0}: K_{1,2}\left(z_{0} ; \tau, \tau^{\prime}\right)$.

\section{Periodic NFT (PNFT)}

The usual NFT operations assume that the optical signal, $q\left(z_{0}, t\right)$, decays as $t \rightarrow \pm \infty$. So the ordinary NFT assumes that we have a burst-mode transmission, Fig. 2, i.e. at each $z=z_{0}$ the signal duration and the processing region coincide. However, in communication applications it is often more convenient to work with periodic signals for the processing of a data stream: The periodicity assumption, which is in our denotations $q\left(z_{0}, t\right)=q\left(z_{0}, t+T_{p}\right)$ for the period $T_{p}$, helps in developing the fast processing procedures, such as the overlapand-save method [82]. Thus, the PNFT may be considered as a natural choice for the replacement of linear (say, FFT-based) processing elements. The PNFT was recently introduced within the circle of available solutions for the nonlinear signal processing in $[83,84]$. Basically, the PNFT offers the same possibilities for the communication system design and concepts (with the use of the periodically-continued signals) as the NFT does for the vanishing signals by adding a cyclic prefix extension instead of zero-padded wings for ordinary NFT, Fig. 2. Together with this, the periodicity assumption can bring about some other benefits. i) Only a finite part of a periodic signal (one period) represents the whole signal, so we do not have to process the entire interval accounting for the dispersion-induced memory, as it occurs for the ordinary NFT, see Fig. 2. Because of this, one can have a considerable processing speed-up when using PNFT. ii) When using an ordinary NFT, in particular, within nonlinear synthesis [51-53], it is difficult to control the time duration of the resulting wave-shapes. Using the PNFT, where signals have a finite "meaningful" time duration (the PNFT period), we can attain more control over the time domain profiles. iii) For the PNFT, the encoding schemes can be, to some extent, based on the encoders of currently used communication systems, as the PNFT share a cyclic-prefix profile extension idea. iv) Producing periodic solutions of NLSE could be generally done using Riemann theta functions that can be seen as the multidimensional generalization of the FT, such that some properties of linear modulation can still be kept within the PNFT paradigm. v) By using a

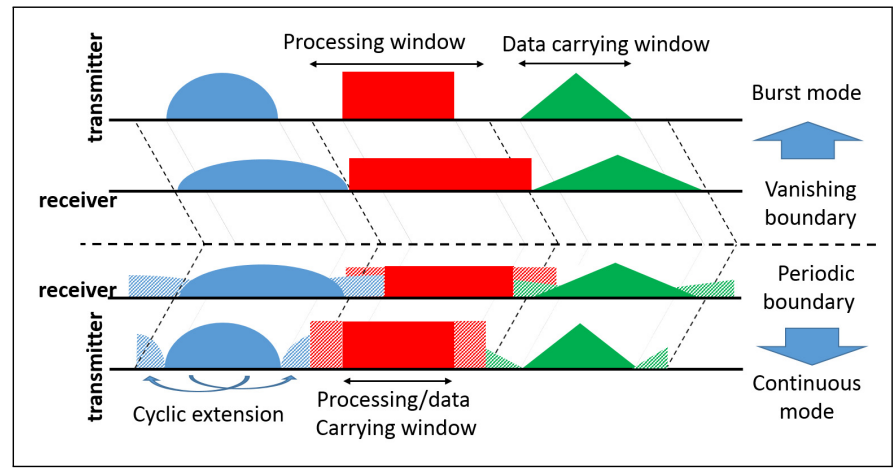

Fig. 2. Burst mode for the window in vanishing signal processing (ordinary NFT) and the processing window for the periodic signal with cyclic extension (PNFT).

periodically-extended signals we can have a continuous stream of data without sudden droppings of power, thus, reducing peak-to-average power ratio (PAPR), in contrast to the burstmode with the ordinary NFT usage. For the sake of completeness we briefly describe below some basic elements of PNFT (see [84-88] for examples of such a communication system).

Direct PNFT. Similarly to the case of ordinary NFT, in the periodic problem we have two parts of nonlinear spectrum associated with a general periodic time-domain profile: The constant main spectrum, which serves as an analog of soliton eigenvalues, and dynamical auxiliary spectrum. In contrast to the ordinary NFT, here both parts of the spectrum consist of discrete points and there is no continuous component. To define the scattering data we now have to deal with the solutions (the so-called Bloch solutions) of ZSP (5) with a periodic potential, $q\left(z_{0}, t\right)=q\left(z_{0}, t+T_{p}\right)$, subject to conditions $\varphi\left(t_{0}, t_{0}, \zeta\right)=[1,0]^{T}$ and $\tilde{\varphi}\left(t_{0}, t_{0}, \zeta\right)=[0,1]^{T}$, where $t_{0}$ is an arbitrary base point. The so-called $2 \times 2$ fundamental matrix is defined through the Bloch ZSP solutions as $\Phi\left(t, t_{0}, \zeta\right)=\left[\varphi\left(t, t_{0}, \zeta\right), \tilde{\varphi}\left(t, t_{0}, \zeta\right)\right]$. Evaluating this fundamental matrix at $t=t_{0}+T_{p}$ one gets the monodromy matrix, $\mathcal{M}\left(t_{0} ; \zeta\right)=\Phi\left(t_{0}+T_{p}, t_{0}, \zeta\right)$. The monodromy matrix plays a crucial role in the Floquet theory which deals with differential systems with periodic structure. At the endpoints of stable bands the Bloch solutions are (anti-)periodic and the values of parameter $\zeta$ corresponding to these endpoints, i.e. the main spectrum, $\mathbb{M}$, can be defined through the Floquet discriminant $\Delta(\zeta)=(1 / 2) \operatorname{Tr} \mathcal{M}\left(t_{0} ; \zeta\right)$ as [83]:

$$
\mathbb{M}=\left\{\zeta_{m}\left|\Delta\left(\zeta_{m}\right)= \pm 1, \frac{d \Delta}{d \zeta}\right|_{\zeta=\zeta_{m}}^{\neq 0}\right\}
$$

A $g$-band (g-gap) periodic solution of NLSE is the solution in which there are only $2 g$ elements in $\mathbb{M}[89,90]$. The important property of the main spectrum is that it remains invariant during the pulse evolution along $z$-direction. The definition of auxiliary spectrum, $\mu_{i}(z, t)$, is given in the Supplementary Material.

Inverse PNFT. The inverse PNFT is the procedure of how to get the (periodic in time) profile $q(z, t)$ starting from given main and (evolved) auxiliary nonlinear spectrum parts. There are several methods to construct finite-gap periodic solutions of NLSE, see [83]. One can use the theta-function representation $[91,92]$ :

$$
q(z, t)=q(0,0) \frac{\Theta\left(\mathbf{W}^{-} \mid \tau\right)}{\Theta\left(\mathbf{W}^{+} \mid \tau\right)} e^{i k_{0} z-i \omega_{0} t}
$$


where $k_{0}$ and $\omega_{0}$ are some constants obtained from the nonlinear spectrum, and the Riemann theta function, $\Theta(\mathbf{W} \mid \tau)$ is defined as [92]:

$$
\Theta(\mathbf{W} \mid \tau)=\sum_{\mathbf{m} \in \mathbb{Z}^{g}} \exp \left(2 \pi i \mathbf{m}^{T} \mathbf{W}+\pi i \mathbf{m}^{T} \tau \mathbf{m}\right) .
$$

Here $\mathbf{m}$ is a vector representing one realization of all permutations of $g$-dimensional arrays with integer elements, and $\mathbf{W}^{ \pm}=$ $\pi\left(\mathbf{k} z+\boldsymbol{\omega} t+\boldsymbol{\delta}^{ \pm}\right) / 2$ is a vector calculated from the nonlinear spectrum. The set $\{\mathbf{k}, \omega, \delta, \tau\}$ is called Riemann spectrum, and $\tau$ is the Riemann (period) matrix [92]; their particular values can be, again, obtained from the full set of nonlinear spectral data. Thus, within the representation (19) the inverse PNFT procedure can be reformulated as the problem of finding the Riemann spectrum from the given nonlinear spectrum.

Although there is still currently a lack of a generic approach for how to deal with the inverse PNFT, there are several software packages allowing one to construct the profile in time domain using the periodic spectral data. For finding the Riemann spectrum, there are some packages and codes embedded in Maple, Sage, and Mathematica [93-95]. For the second stage, which is to construct the Riemann theta functions (20) using the Riemann spectrum, in addition to the symbolic implementations [96, 97], some "hyper-fast" algorithms for the numerical reconstruction of special classes of signals were proposed [92].

\section{NUMERICAL ALGORITHMS FOR THE NFT}

In this section, we overview existing numerical methods for the forward (5) and inverse (16) NFT, paying particular attention for the methods that have already been tested for the transmission purposes. The goal of the forward NFT is to calculate the nonlinear spectrum $\Sigma\left(z_{0}\right)$ (13) from the given space-time domain profile $q\left(z_{0}, t\right)$. The INFT method must provide the timedomain waveform starting from given $\Sigma$.

The signal $q\left(z_{0}, t\right)$ is in practice only known at the specific points in time due to sampling operations, which means that for the forward NFT the nonlinear spectrum has to be approximated based on the samples

$$
q_{m}=q\left(z_{0}, T_{1}+\epsilon m\right), \quad m=0,1, \ldots, M-1,
$$

where $T_{1}$ is close enough to $-\infty$ such that the boundary condition in Eq. (6) is approximately satisfied for $t=T_{1}$ and $T_{2}>T_{1}$ is sufficiently close to $+\infty$ such that Eq. (10) is approximately satisfied, respectively. The parameter $\epsilon=\left(T_{2}-T_{1}\right) /(M-1)$ denotes the sampling interval.

The methods are classified according to how their numerical complexity (in terms of floating point operations, flops) and the accuracy of the result changes as the number of sample points $M$ increases.

\section{A. Algorithms for direct NFT}

Numerous algorithms for computing the NFT have been described in the literature. The two best known are probably the methods of Ablowitz-Ladik and Boffetta-Osborne. We will first describe these two methods and then briefly list other approaches. More details can be found in [45, 83, 98]. We, however, note that our review does not, of course, cover all existing possibilities for the NFT operations implementation (e.g. in recent work [99] a bi-direction algorithm for the calculation of soliton norming constants was described) and we rather concentrate on the methods that have already found their way into optical transmission studies, although there are some new methods which have yet to be tested, see e.g. [100].
Boffetta-Osborne (BO) transfer method. The general idea is to approximate the Jost scattering functions $a(\zeta)$ and $b(\zeta)$ using Eq. (10). Therefore, Boffetta and Osborne [101] assumed that the signal $q\left(z_{0}, t\right)$ is piecewise constant, i.e. $q\left(z_{0}, t\right)=q_{m}=$ const. for $t \in\left[T_{1}+(m-0.5) \epsilon, T 1+(m+0.5) \epsilon\right)$ and solved the ZSP (5) in closed form under that assumption. For each interval $\left[T_{1}+(m-0.5) \epsilon, T 1+(m+0.5) \epsilon\right)$, one has

$$
\begin{aligned}
{\left[\begin{array}{l}
\phi_{1}\left(T_{1}+(m+0.5) \epsilon, \zeta\right) \\
\phi_{2}\left(T_{1}+(m+0.5) \epsilon, \zeta\right)
\end{array}\right] } & =T_{m}\left[\begin{array}{l}
\phi_{1}\left(T_{1}+(m-0.5) \epsilon, \zeta\right) \\
\phi_{2}\left(T_{1}+(m-0.5) \epsilon, \zeta\right)
\end{array}\right] \\
\text { where } T_{m}(\zeta) & =\exp _{m}\left(\left[\begin{array}{cc}
-i \zeta & \epsilon q_{m} \\
\mp \epsilon \bar{q}_{m} & i \zeta
\end{array}\right]\right)
\end{aligned}
$$

Here, $\exp _{m}(\cdot)$ denotes the matrix exponential. Taking the boundary conditions (6), (10) into account, one finds that

$$
\left[\begin{array}{l}
a(\zeta) \\
b(\zeta)
\end{array}\right] \approx e^{i \zeta\left(T_{2}+0.5 \epsilon\right)} T_{M}(\zeta) \times \cdots \times T_{1}(\zeta)\left[\begin{array}{c}
e^{i \zeta\left(T_{1}-0.5 \epsilon\right)} \\
0
\end{array}\right] .
$$

This approximation can be used straight-away to evaluate the RC Eq. (11). In order to locate the discrete eigenvalues $\zeta_{n}$, Boffetta and Osborne proposed to apply Newton's method to $a(\zeta)$. A nonlinear version of Parseval's relation can be used to check if all discrete eigenvalues have been found [101]. The complexity for evaluating Eq. (23) in a straight-forward way is $O(M)$. The total complexity of a search method to find the discrete eigenvalues is therefore $O\left(k_{\text {iter }} N_{\text {guesses }} M\right)$, where $k_{\text {iter }}$ is the average number of iterations per initial guess and $N_{\text {guesses }}$ is the number of initial guesses used. The complexity of evaluating the RC Eq. (11) on a grid of $M$ nonlinear frequencies is $O\left(M^{2}\right)$.

The $\mathrm{BO}$ method has a second order approximation accuracy, i.e., for any fixed $\zeta=\zeta_{0}$, the distance between the numerical approximations of $a\left(\zeta_{0}\right)$ and $b\left(\zeta_{0}\right)$ and their true values is of the order $O\left(M^{-2}\right)[101,102]$. Note that the hidden constant in the big-O notation depends on $\zeta$. For the BO method, the hidden constant was found to be $\sim|\zeta|^{-1}$ for large $\zeta$ in [102]. The BO method was used in the works $[49,51-53]$ for the calculation of continuous nonlinear spectrum for the nonlinear inverse synthesis scheme (see subsection 5B below). It also demonstrated good results in the calculation of the perturbed dynamics of solitonic eigenvalues [102-104]. The calculation of norming constants, requiring $a^{\prime}\left(\zeta_{n}\right)$, is described in [101, 102]. In [102], the BO method was compared to the direct 4 th order Runge-Kutta integration of the ZSP (5), where for the latter method the hidden constant in the big-O notation was found to be $\sim|\zeta|^{4}$. It was concluded that, generally, the BO method is more convenient especially when the wide range of $\zeta$ values is addressed.

Ablowitz-Ladik (AL) discretization method (normalized). The AL discretization $[105,106]$ is another method widely used for the NFT-based transmission [60-65]. It corresponds to the approximation of NLSE by a discrete integrable problem. The method also takes the form in Eq. (23), but with

$$
T_{m}(\zeta)=\frac{1}{\sqrt{1 \pm \epsilon^{2}\left|q_{m}\right|^{2}}}\left[\begin{array}{cc}
Z & \epsilon q_{m} \\
\mp \epsilon \bar{q}_{m} & Z^{-1}
\end{array}\right], \quad Z=e^{i \zeta \epsilon}
$$

For the location of zeros of $a(\zeta)$ one again has to apply an additional search routine. It was shown [45] that the AL algorithm can produce some small spurious solitonic eigenvalues which, however, can be readily sorted out. In Ref. [56] the AL 
method was compared with the BO method for realistic NFTbased transmission parameters, and it turned out that the AL method demonstrates a slightly better performance when applied to the calculation of continuous spectrum (RC). The accuracy of the AL algorithm is of the second order as for the $\mathrm{BO}$ scheme. This was e.g. shown in [107] for a variant of the AL algorithm that is commonly used in fiber Bragg gratings design. The relation of the scheme in [107] to the AL algorithm below is elaborated in [108]. Apparently in contrast to this, Boffetta and Osborne had observed in [101, 109] that the AL discretization achieves only a first order accuracy, when the discrete eigenvalues computed by the $\mathrm{AL}$ and $\mathrm{BO}$ methods were compared to exact analytical values. The AL discretization that was investigated in [109] however was an early version [105], in which the coordinate transform $Z=1-i \zeta \epsilon$ was used instead of the now common transform $Z=e^{i \zeta \epsilon}$ that was given later in [106]. In various numerical experiments that were reported in [45], the errors of the AL and $\mathrm{BO}$ schemes decrease at similar rates w.r.t. $M$. The same inference was confirmed in the study [56], related to the true NFT-based transmission profiles.

Fourier collocation method has been used by the Osaka group and co-authors [66-72]. Within this method the ZSP solution components $v_{1,2}$ are expanded in the Fourier series and the ZSP itself is reformulated as an eigenvalue problem in the Fourier space [43, 45]. However this method is inconvenient for the computation of the continuous nonlinear spectrum and soliton norming constants, and it has been used only for the eigenvalue communication where the solitonic discrete eigenvalues themselves are adopted as information carriers. Another drawback of this method is its the numerical complexity: The method requires the diagonalization of non-Hermitial (for anomalous dispersion) matrix, where the number of required flops is $O\left(M^{3}\right)$.

Direct Toeplitz inner bordering (TIB) method. A new efficacious algorithm for the computation of continuous nonlinear spectrum using the Toeplitz matrix transformations, was proposed in [110]. In the numerical example considered in [110] TB outperforms the BO method in terms of speed and accuracy, it has the error level of $O\left(M^{-2}\right)$ and the number of flops $O\left(M^{2}\right)$. This method is based on the reversion of the Toeplitz matrix-based INFT algorithm; we provide the corresponding INFT in the next subsection and description of the method in Supplementary Material. However, when dealing with the direct TIB method, one has to keep in mind that it recovers the kernel of the GLME $L(\tau)$ that in general includes both discrete (solitonic) and continuous spectral components, Eq. (17), simultaneously.

\section{B. Numerical methods for the INFT}

The methods for numerical INFT computation were largely studied within the Bragg gratings synthesis and characterization. Here, using the traditional "matrix-inversion" terminology, we name the INFT methods requiring $O\left(M^{2}\right)$ operation as "fast", and those with the lower complexity as "superfast". Almost all INFT approaches are based on the numerical solution of GLME (16). After the discretization, one aims at determining functions $K_{1,2}\left(\tau, \tau^{\prime}\right)$ on the grid of $M \times M$ points. Note that the straightforward path there based on the solution of $M$ nested linear matrix equations takes $O\left(M^{4}\right)$ flops is unproductive.

The earlier approaches utilize iterative methods of matrix inversion with the computational complexity in the order of $O\left(k_{i} M^{3}\right)$, where $k_{i}<M$ is a number of iterations. As an example, we mention the group of methods with the GLME kernel parametrization [111, 112]. A similar method was also employed recently for optical transmission tasks [58]. The main drawbacks of these algorithms are the problem of choosing an initial approximation and high computational complexity. A more advanced family of algorithms is based on the layer peeling (LP) method. This class of methods is built on the representation of the RC attributed to a particular profile $q(t)$ through the sequence of individual actions of $M$ point reflectors [79, 80, 113]. The LP algorithms are comparatively fast and require about $O\left(M^{2}\right)$ flops. Some of them provide an error that is globally proportional to $M^{-2}$. Conventional algorithms based on LP show numerical instabilities with exponential amplification of noise when the reflection coefficients that are provided to the LP step are not entirely realizable [114]. This means that there exists no signal such that forward scattering with $M$ samples can result in the desired reflection coefficient [107, 115], or, in other words, when one has independent noisy additions to the scattering data themselves. Physically, when some profile corrupted by noise in the space-time domain is converted into the NF spectrum, and then this spectrum is used for the superfast LP algorithm considered further, we observed that the instability in all numerical examples was absent, see Supplementary Material. For the properties of space-time noise conversion into the NF domain see Refs. [116-119]. In some transmission systems this limitation has recently been circumvented in some first algorithms where the reflection coefficient is ensured to be realizable by construction $[73,108,120]$. On the other hand, this instability can reveal itself when one synthesizes a profile starting from some randomly encoded spectral data in the NFD, and this question requires further analysis.

The LP with improved accuracy [81], known as an integral LP has some issues with the overall efficiency, as it requires much more arithmetic operations. The drawback of these algorithms is the accumulation of computational errors during calculation and the resulting decrease in their accuracy when enlarging the $q(t)$ extent.

Another interesting group of algorithms are based on recasting the GLME as the system of partial differential equations [121, 122] (see also [123] for the comparison of such algorithms), including also the "leap-frog" algorithm [124]. The numerical complexity is $O\left(M^{2}\right)$ flops; however, the error there is only of the 1st order, $O\left(M^{-1}\right)$ [122, Fig. 2 b].

In [125] another algorithm was proposed, based on a different computational approach, whose error was proportional to $M^{-2}$. This algorithm is "slow", requiring $O\left(M^{3}\right)$ flops, and addresses only the case of normal dispersion. However, the important feature there is that it introduced the very idea of the bordering procedure itself. Later, a more efficient algorithm that has a $M^{-2}$ error and at the same time uses $O\left(M^{2}\right)$ flops was described in [110, 126]. The algorithm exploits the Toeplitz symmetry of discretized GLME using TIB, similar to technique for common Toeplitz matrices [127, 128]. As the TIB was successfully used in a number of transmission-related works [51-55], we provide here more details on TIB method. First, we change the variables in (16) as

$$
u\left(\tau, \tau^{\prime}\right)=K_{1}\left(\tau, \tau-\tau^{\prime}\right), \quad w\left(\tau, \tau^{\prime}\right)=\mp \bar{K}_{2}\left(\tau, \tau^{\prime}-\tau\right) .
$$

In new notations, explicitly assuming the finite extent of $q(t)$, $0 \leq t \leq T$, after the complex conjugation of the first of GLME, 
we get:

$$
\begin{aligned}
& u(\tau, y) \mp \int_{y}^{2 \tau} \bar{L}\left(\tau^{\prime}-y\right) w\left(\tau, \tau^{\prime}\right) d \tau^{\prime}=0, \\
& w\left(\tau, \tau^{\prime}\right)+\int_{0}^{\tau^{\prime}} L\left(\tau^{\prime}-y\right) u(\tau, y) d y+L\left(\tau^{\prime}\right)=0,
\end{aligned}
$$

$0 \leqslant y, \tau^{\prime}<2 \tau, 0 \leqslant \tau \leqslant T$. The sought solution in time domain now reads as $q(t)=2 w(t, 2 t-0)$. The GLME form (26) allows one to to obtain the Toeplitz-type problem after the discretization and to use the fast Toeplitz matrix inversion algorithms $[127,128]$ for the recovery of $q(t)$. Further details of the TIB-based INFT are given in Supplementary Material.

At the end we mention a recent work on the INFT methods by Civelli et al. [129]: the authors introduced yet another INFT first order solution algorithm based on iterated convolutions with the GLME kernel using the FFT, which demonstrated the better performance in terms of accuracy and time consumption that the 1st order TIB [52] and the Nyström conjugate gradient method [130]. However, the last approach has not been tested so far on the transmission-related problems. Note that NFT can be formulated in terms of the so-called Riemann-Hilbert problem (see e.g, [43] and references therein) and numerical solution of NFT can be implemented using this approach [131].

\section{Superfast NFT algorithms}

It has recently been observed that the AL method (and others) for computing the NFT can be significantly sped up, leading to a superfast NFT analogous to the celebrated FFT [83, 98]. We illustrate hoe to deal with fast NFTs using AL discretization.

The matrix $T_{m}(\zeta)$ in Eq. (24) can be written as

$$
T_{m}(\zeta)=S_{m}(Z) / d_{m}(Z), \quad Z=e^{i \lambda \epsilon},
$$

where $S_{m}(Z)$ and $d_{m}(Z)$ are polynomials with respect to $Z$ :

$$
S_{m}(Z)=\frac{1}{\sqrt{1+\epsilon^{2}\left|q_{m}\right|^{2}}}\left[\begin{array}{cc}
Z^{2} & \epsilon q_{m} Z \\
-\epsilon \bar{q}_{m} Z & 1
\end{array}\right], \quad d_{m}(Z)=Z .
$$

Consequently, with $S(Z)=S_{M}(Z) \times \cdots \times S_{1}(Z)$ and $d(Z)=$ $d_{M}(Z) \times \cdots \times d_{1}(Z)$, Eq. (23) can be written as

$$
\left[\begin{array}{l}
a(\zeta) \\
b(\zeta)
\end{array}\right] \approx e^{i \zeta\left(T_{2}+0.5 \epsilon\right)} \frac{S(Z)}{d(Z)}\left[\begin{array}{c}
e^{i \zeta\left(T_{1}-0.5 \epsilon\right)} \\
0
\end{array}\right] .
$$

Since the $S_{m}(Z)$ and $d_{m}(Z)$ are polynomials with degrees at most two, both $S(Z)$ and $d(Z)$ are again polynomials whose degrees are upper bounded by $2 M$. The superfast NFT exploits this observation and proceeds in two steps. First, the monomial expansions of the polynomials $S(Z)$ and $d(Z)$ have to be computed. That is, the unique matrices $S^{(k)}$ and scalars $d^{(k)}$ need to be found such that

$$
S(Z)=\sum_{k=0}^{2 M-1} S^{(k)} Z^{k}, \quad d(Z)=\sum_{k=0}^{2 M-1} d^{(k)} Z^{k} .
$$

One first needs a fast method to compute the monomial expansions. A naive implementation, e.g. to compute the expansion of $S(Z)$, would proceed as follows,

$$
S(Z)=S_{M}(Z)\left[S_{M-1}(Z)\left[S_{M-2}(Z)\left[\cdots\left[S_{1}(Z)\right]\right]\right] \ldots\right] .
$$

However, this leads to a $O\left(M^{2}\right)$ or even $O\left(M^{3}\right)$ runtime, depending on how the product of polynomials is found. In order to get a superfast NFT algorithm, a divide-and-conquer strategy is used instead. One starts with the elementary polynomials $S_{m}(Z), m=0, \ldots, M-1$, partitions them into pairs and computes the products of these pairs. The products are again partitioned into pairs, and then multiplied. This process is iterated until only one product is left, which will be $S(Z)$. It turns out that this algorithm finds the monomial expansion $S^{(k)}$, $k=0, \ldots, M-1$ using only $O\left(M \log ^{2} M\right)$ flops given that polynomial products are computed with the FFT. The pseudocode for this algorithm is provided in Supplementary Material.

The second step of the superfast NFT now applies algorithms for fast polynomial arithmetic in order to compute the NFT spectrum. To approximate the RC in Eq. (11) on an equidistant grid $\lambda_{m}=\lambda_{1}+m \delta$, where $\delta=\left(\lambda_{2}-\lambda_{1} /(M-1)\right.$ and $m=0,1, \ldots, M-1$, one needs to evaluate the polynomials $S(Z)$ and $d(Z)$ at the points

$$
Z_{m}=e^{i\left(\lambda_{1}+m \epsilon\right)}=e^{i \lambda_{1}}\left(e^{i \epsilon}\right)^{m}=a w^{m} .
$$

The chirp transform algorithm [132] thus allows us to compute $S\left(Z_{1}\right), \ldots, S\left(Z_{M}\right)$ as well as $\left.d\left(Z_{1}\right), \ldots, d\left(Z_{M}\right)\right)$ using only $O(M \log M)$ flops. The discrete-eigenvalues $\zeta_{m}$, which are the roots of $a(\zeta)$ with positive imaginary part, can be found quickly by using a class of recently developed root-finding algorithms. These algorithms implement the well-known idea to find the roots of a polynomial from the eigenvalues of a so-called companion matrix. But while a conventional eigenvalue finder will require $O\left(M^{3}\right)$ flops, the algorithms e.g. in [133, 134] manage to exploit the structure of the companion matrix such that the runtime is reduced to $O\left(M^{2}\right)$ flops. We remark that fast polynomial arithmetic can also be used to speed-up Newton's method for finding the discrete spectrum. A first concept for a $O\left(k_{\text {iter }} M \log ^{2} M\right)$ algorithm has recently been presented [135].

In summary, the superfast NFT discussed here can compute the RC using $O\left(M \log ^{2} M\right)$ flops and the discrete eigenvalues using $O\left(M^{2}\right)$ flops. The conventional methods discussed in Subsection A require, in comparison, $O\left(M^{2}\right)$ and $O\left(k_{\text {iter }} N_{\text {guesses }} M\right)$ flops, respectively. The algorithm in this section is thus clearly faster for the RCs, and it was found in a recent numerical study [56] that it outperformed a conventional, but parallelized implementation of the $\mathrm{BO}$ algorithm that ran on a dedicated GPU both in terms of quality and runtime. It is harder to compare the complexities of computing the discrete eigenvalues in a communication scenario where the constellation of discrete eigenvalues is drawn from some modulation alphabet, see [98]. A detailed numerical study of these issues in a communication scenario is still to be performed. In any case, it should be noted that even in cases without speed-up, the method discussed in this section does not require any tuning. This is in contrast to search methods such as in [45], which require the use to choose several parameters that can have a large influence on both runtime and accuracy.

\section{Superfast INFT algorithm}

The superfast NFT algorithm that was discussed in Subsection $\mathrm{C}$ for the AL discretization proceeds in two steps: 1) Compute the rational approximations $a(\zeta) \approx e^{i \zeta(T 2-T 1+\epsilon)} S_{11}(\zeta) / d(\zeta)$ and $b(\zeta) \approx e^{i \zeta(T 2-T 1+\epsilon)} S_{21}(\zeta) / d(\zeta)$ in a fast manner, and 2$)$ compute the NFT spectrum from these approximations using fast algorithms for polynomial operations. In order to obtain superfast inverse NFT algorithms, the idea of reversing these two steps was proposed in $[108,120]$. The two steps of a superfast inverse NFT method using this idea are thus: 1) Deter- 
mine polynomials $S(\zeta)$ and $d(\zeta)$ fast such that Eq. (29) leads to a good approximation of a given nonlinear Fourier spectrum, and 2) compute the samples of the corresponding time-domain signal $q_{m}$ fast by exploiting Eq. (23).

For the first step, two superfast methods that can be used to generate multi-solitons have been presented in [120, 136]. Furthermore, a superfast method that solves the first step for signals with empty discrete spectrum has been proposed in [137]. By combining the results in [136] and [137], a general method for arbitrary spectra with a complexity of $O(M N+$ $\left.M \log ^{2} M\right)$ has finally been obtained in [138]. The difficulty in the first step is that the polynomials $S(\zeta)$ and $d(\zeta)$ cannot be chosen arbitrarily. Similar to the continuous-time case, where $|a(\zeta)|^{2} \pm|b(\zeta)|^{2}=1$ for all real $\zeta$, the generated polynomials $S(Z)$ and $d(Z)$ have to satisfy $\left|S_{11}(Z)\right|^{2} \pm\left|S_{21}(Z)\right|^{2}=|d(Z)|^{2}$ whenever $|Z|=1$. If this condition is not fulfilled, we have $S(Z) \neq S_{M}(Z) \times \cdots \times S_{1}(Z)$ no matter how the $q_{m}$ are chosen. In other words, the second step of recovering the $q_{m}$ through Eq. (23) becomes ill-posed.

The second step of recovering the samples $q_{m}$ from the polynomials $S(Z)$ and $d(Z)$ can be performed efficiently using only $O\left(M \log ^{2} M\right)$ flops with a technique that was developed in the area of geophysical prospecting by McClary [139], and has been adapted for the computation of INFT in [120]. Further details are given in Supplementary Material.

\section{NFT FOR OPTICAL COMMUNICATIONS}

As the evolution of nonlinear spectrum inside the nonlinear Fourier domain (NFD) is linear and decoupled, the signal's NF spectrum can be efficaciously used for coding, transmission, detection and processing of information. Though all recent works on the NFT can be deemed of as the modifications and extensions of the original idea proposed in [42], in works [44-46] the pertinent new term "nonlinear frequency division multiplexing" (NFDM) was introduced to stress the analogy to OFDM. However, in the following, we will use the umbrella term "Modulation in the NFD" to refer to this type of schemes where the quantities from the NF domain are used as the information carriers. There are three basic designs for NFT-based transmission systems which are schematically presented in the Figs. 3, 4. In the first design, the transmitted information is encoded directly onto the NF signal spectrum via the INFT: It is "Modulation in the NFD". Within this design, one can modulate either discrete [60] and continuous [51, 52] NF spectrum parts separately or simultaneously [58]. In the second design, the NFTs are used to cancel the nonlinear distortions at the receiver. This scheme can be understood as the DBP with the use of the NFT operations, NFT-DBP [50, 73]. Here, the signal encoding and modulation is performed in a similar way to the conventional transmission systems. However, one critical challenge in the NFT-based DBP is the requirement of the accurate calculation of an unknown number of discrete eigenvalues in the randomly coded information-bearing signal. As a result, so far the NFTbased DBP approach was elaborated only for the soliton-free case [50,73]. For the third design, which is referred to as the "hybrid method", information is encoded in the time domain but the detection stage involves NFT operations and decision is made using NF spectrum data $[62,63,66,67,70]$.

\section{A. Modulating the discrete part of NF spectrum}

Recently, the data transmission using discrete eigenvalues (solitonic) components of the signal's nonlinear spectrum has been

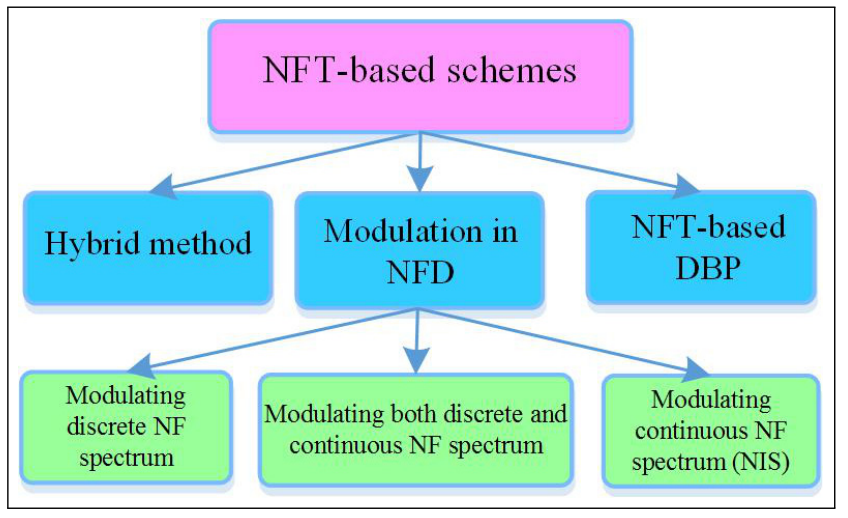

Fig. 3. Diagram of the currently proposed and studied NFTbased methods.

intensively studied theoretically and experimentally [46, 60, $61,65,99,140-142]$. Within this approach, one starts with a predefined set of discrete eigenvalues and related parameters (discrete constellation in the NFD), onto which the information is mapped. This step can be referred as constellation design, where the positions of discrete eigenvalues in the complex plane and norming constants are optimized for maximizing the system performance and SE. Next, the corresponding time-domain waveforms are generated using the INFT (e.g. by using Darboux method, see Supplementary Material). At the receiver, NFT is applied to recover modulated discrete eigenvalues and norming constants.

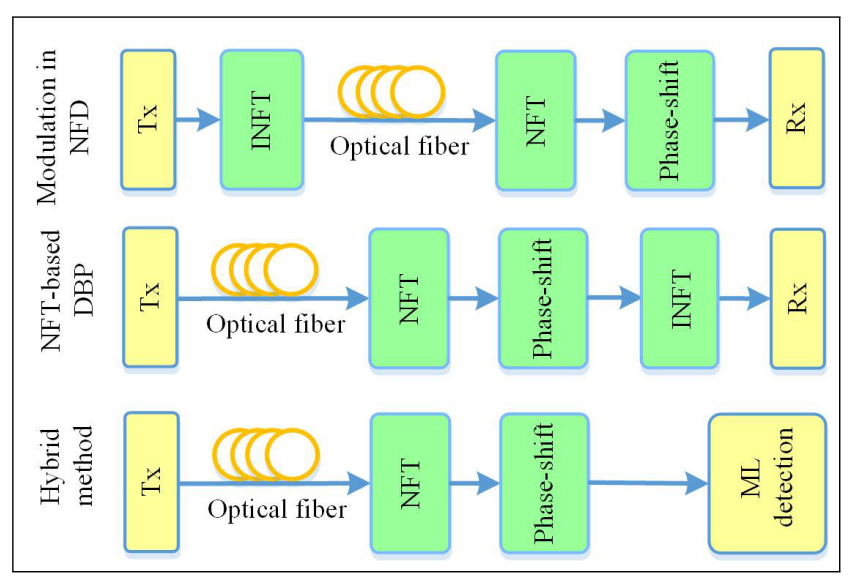

Fig. 4. Basic designs of NFT-based transmission systems, including transmission in the NFT domain, DBP with the use of NFT operations, and the hybrid method.

\section{B. Modulating the continuous NF spectrum. Nonlinear in- verse synthesis (NIS)}

The NIS scheme, which is based on the modulation of the continuous part of the signal nonlinear spectrum has been proposed recently [51], for the anomalous dispersion case. This scheme has a good potential as it exploits the vast amount of available degrees of freedom contained in the continuous part of the nonlinear spectrum. At the same time, it also allows one to avoid the problems associated with the solitonic degrees of freedom. Within this scheme, the linear spectrum of an input signal is first mapped onto the continuous part of the nonlinear 
spectrum of a complex field (to be transmitted) via the INFT. This step makes it possible to translate directly a standard modulation format into the nonlinear spectral domain. At the receiver, the nonlinear spectrum of the transmitted field is obtained using the NFT and then a single-tap linear dispersion removal is applied to remove the deterministic nonlinear impairments. Since the input field of the INFT can be arbitrary, high order modulation formats can be combined with the NIS scheme, providing the flexibility in the system's design [52]. It has been shown in [52] that the NIS scheme can provide a performance gain up to $4.5 \mathrm{~dB}$ compared to the linear compensation when combined with 64 QAM modulation format. Furthermore, the NIS scheme can also be applied in optical links with EDFA and non-ideal DRA by employing the LPA NLSE model [53-55].

One major advantage of the NIS scheme, as of a DSP-based approach, is that it can be easily integrated with the current coherent transmission technology [57]. In addition, the numerical complexity of NIS can be competitive and potentially even outperform that of the standard DBP based on the split-step NLSE backward solution with the recent advances in NFT algorithms $[83,98]$. The first successful integration of the superfast NFT processing into the NIS scheme was reported in [56]. It has to be noted that at the moment the SE of the NIS system, which is built on the continuous NF data, is relatively low because, in current implementations, the extend of the fiber-input usually exceeds that of the initial waveform due to slowly decaying wings. Another issue is that it currently can be difficult to generate long symbols with the INFT because this requires an improved floating point precision (see the remark in subsection C). This is, however, an early stage of the NIS method, and we anticipate that the SE will improve with further optimization.

In the very recent works $[58,59]$ first demonstrations that the NIS approach can be combined with solitons have been given, both numerically and experimentally. This paves the way for the design of advanced combined NFDM-NIS modulation schemes, where all available degrees of freedom inside the NFD are utilized for the modulation and transmission.

\section{NFT-based DBP}

Nonlinear and dispersive transmission impairments in coherent fiber-optic communication systems are often compensated by reverting the NLSE numerically. This technique is known as DBP [18]. Typical DBP algorithms are based on split-step Fourier methods in which the signal has to be discretized in time and space. The need to discretize in both time and space however makes the real-time implementation of DBP a challenging problem. The NFT technique offers a different fast algorithm for the DBP [73]. The method itself was first introduced in Ref. [50]. Since the spatial evolution of a signal governed by the NLSE can be reverted analytically in the NFT domain through simple phase-shifts, there is no need to discretize the spatial domain. The superfast algorithm described above can be used to process signals in the time domain and is therefore highly promising for real-time implementations. However, we note again that both NFT-DBP works mentioned above deal with the normal dispersion case, and the NFT-based DBP for the anomalous dispersion has yet to be deployed. The performance of NFT-DBP has been observed to degrade for symbols with long durations and / or high powers due to numerical problems [143]. We expect that this can be addressed by increasing the precision (and complexity) of the floating point operations.

\section{Hybrid method. MD detection inside the NFD}

Within this approach, one starts with modulating (predefined) waveforms in the time domain, for which the corresponding discrete eigenvalues are known $[66-68,71]$ or easily calculated [62-64]. The transmitted information is mapped onto these waveforms. At the receiver, decisions are made through the NFT processing based on the detected discrete eigenvalues [66$68,71]$ or using both the eigenvalues and the norming constant [62-64]. As this approach does not offer the flexibility of optimizing the positions of discrete eigenvalues in the complex plane, a minimum distance (MD) detector is usually required. In addition, if the transmitted waveforms are not optimized for multi-soliton transmissions, the continuous part of the signal's nonlinear spectrum should also be taken into account $[62,63]$.

\section{EFFICIENCY ESTIMATES FOR NFT-BASED TRANS- MISSION}

There is currently a widespread belief that the nonlinear optical channel capacity is still largely unknown: As discussed in [146], [147], exact channel capacity results for fibre optical systems are scarce, and many aspects related to this problem remain open. The transmission methods employing on-off-keying (OOK) fundamental solitons have been almost abandoned, mostly due to the low $\mathrm{SE} \approx 0.2 \mathrm{bits} / \mathrm{s} / \mathrm{Hz}$ of $\mathrm{OOK}$ soliton systems limited by the celebrated Gordon-Haus jitter phenomena [148], and, in addition, due to the problems with inter-soliton interaction between the WDM soliton channels [27]. However, the NFT methods are much richer than the soliton OOK, and there is a reason to expect drastically different results for the SE when NFT is used in its full scale. Together with this, due to the complexity allowed in the contemporary receivers, one can address the question of NFT channel capacity for quite involved modulation schemes, nontrivially-defined signal space, and sophisticated error correcting codes. However, the SE and capacity problems formulated for the NFD channels are quite new, and not so many results are available at the moment. Meron et al. [76] were, probably, the first who recognised that the mutual information in a nonlinear integrable channel (the NLSE was considered) can be evaluated through the statistics of the NFD data, i.e., via the channel defined inside the NFD: The mutual information between the input and output waveforms is equivalent to the mutual information between the input and output NFT spectra. In work [76] the authors presented the data for the lower bound of mutual information and capacity per channel use of soliton-based system (the imaginary parts of the multiply eigenvalues were modulated), using the Gaussian scalar model for the amplitude evolution perturbed by the progenitor in-line noise. They showed that for a single soliton continuous amplitude modulation the capacity 1.568 bits/channel is achievable. Then the bit rate gain due to the continuous modulation against the OOK soliton system was analysed for a single, two and more solitons (well-separated soliton trains were considered) per one time slot, with the account of Gordon-Haus jitter [27, 34, 148], showing approximately a factor 2 bit rate improvement vs. OOK. In the following work [46], Yousefi and Kschischang provided results for the lower bounds of achievable SE and bit rates for the modulation of either discrete or continuous parts of NFT spectrum. We note that the norming constants modulation and non-trivial eigenvalue constellation point positioning were also considered there, and the results were again compared with those pertaining to the OOK systems. The 0.4 bits $/ \mathrm{s} / \mathrm{Hz}$ lower bound SE was reported for 
4-point eigenvalue modulation, and for more sophisticated simultaneous eigenvalue and norming constant modulation with 16 points in the constellation, the SE $\approx 0.73 \mathrm{bits} / \mathrm{s} / \mathrm{Hz}$ was reached; for the 6 eigenvalues and 30 constellation points the $\mathrm{SE}$ of $1.5 \mathrm{bits} / \mathrm{s} / \mathrm{Hz}$ was finally reported. Noticeably that some first data on the continuous NFT spectrum modulation based on raised cosines were also given in that study: The maximum $\mathrm{SE}$ of 8 bits/channel and 7 bits/channel were reported for the single-channel and WDM transmission inside the NFD, correspondingly. Developing the idea of multieigenvalue communication, Hari et al. [60] demonstrated the SE of 3.14 bits $/ \mathrm{s} / \mathrm{Hz}$, by employing the modulation of 5 imaginary eigenvalues and using exhaustive search for optimal time-duration and bandwidth of the resulting pulses. In [99] the SE of 3 bits/s/Hz was reported for simultaneous modulation of norming constants and amplitudes of a single soliton at $2000 \mathrm{~km}$; for the 2-soliton system in the case of ignoring the intercomponent interaction, the SE of 1.8 bits/s/Hz was reported. Generally, it was inferred that the SE of a 1-soliton system is higher than that of a 2-soliton one. In work [140] two heuristic designs for multisoliton signal sets are described, and the spectral efficiencies reported somewhat exceed $3 \mathrm{bits} / \mathrm{s} / \mathrm{Hz}$. However the authors noted that the multisoliton signals are significantly limited by bandwidth expansion if the system length is not much smaller than the dispersion length, such that modulating the eigenvalues alone cannot address the problem of nonlinearity in commercial fiber transmission systems and the proposed modulation methods are efficient when dispersion is dominated by nonlinearity. Multieigenvalue communication with all 4 parameters of each soliton modulated was also addressed in [149]: Analytical expressions for the lower bounds for the joint mutual information were derived and achievable rate (in Gaussian approximation) was obtained by using the uniform input distribution subject to the peak constraints. Shevchenko et al. in [77] studied a non-Gaussian (the noncentral $\chi$ distribution, derived in $[150,151])$ model for the noise-perturbed single soliton amplitude evolution addressing the continuous modulation inside the NFD associated with NLSE, showing that the lower bound for the capacity per channel use of such a model is a unbounded growing function of the effective SNR. This result was very recently generalized to the case of Manakov soliton amplitude modulation [153] based on the non-central $\chi$-distribution for noise-perturbed the Manakov soliton amplitude [154]. In [155], the authors addressed the question of capacity and spectral efficiency per symbol for the NIS-based transmission (based on continuous spectrum modulation), using the channel model obtained within nonlinear continuous spectrum perturbation theory [156, 157]: Using very conservative Pinsker estimates for the lower capacity bound [6, 158], it was shown that the estimates for the lower bound for the capacity per symbol of NIS-based transmission are $\approx 10.7$ bits/symbol for $5 \times 100$ GHz WDM Nyquist and OFDM transmission at $2000 \mathrm{~km}$; This bound improves logarithmically with the channel bandwidth or subcarrier spacing. A very similar result has been recently obtained by Yousefi and Yangzhang in the case of normal dispersion [159]: By using direct simulations they demonstrated that the symbol rate for the raised cosine-based WDM inside the NFD is 10.5 bits/symbol at $2000 \mathrm{~km}$, which translates into the SE value of 1.54 bits $/ \mathrm{s} / \mathrm{Hz}$.

\section{CONCLUSION}

In our review, we have classified the existing approaches and methods in the rapidly growing area of NFT-based optical communications under a common framework. The NFT is a truly nonlinear method, that paves the way to the development of fundamentally new, specifically nonlinear techniques for coding, modulation, transmission, and processing of signal in nonlinear communication channels. In spite of some evident success and, currently, numerous experimental demonstration of various types of the NFT-based transmission, this approach still requires considerable efforts directed towards its optimization with respect to reaching higher SE and quality of transmission. Another important way of further NFT methods development refers to the usage of the whole nonlinear domain altogether and the design of the advanced combined methods, like NFDMNIS, to get the improved transmission performance. In addition, the advanced algorithms like the superfast NFT, should be widely implemented and tested for different NFT transmission variants: the existing NFT methods imply either one or two NFT operations, and so the superfast NFT processing algorithms can greatly help in reducing the overall processing time consumption to several orders compared to other methods, the problem which is currently considered as one of the main challenges in the DSP. Finally, we express our sincere hope that the general ideas of NFT and the specifically nonlinear signal characteristics, such as the nonlinear spectrum, will become no less common and routine for optical engineers than the ordinary Fourier operations and corresponding processing methods are now. However, this still requires coordinated efforts from the communication engineers and nonlinear physics communities.

Future key challenges. Finally, we present a list of key challenges we think future research should address. Despite many research efforts and positive progress in recent years, the expected performance and achievable SE of NFT based transmission systems have not been achieved so far, especially experimentally. i) One major challenge in implementing NFT based systems is the implementation penalty due to limitations of practical devices, including linear and nonlinear responses, phase noise [160] and limited resolutions of current available optical transceivers. Application of conventional modulation formats for NFT-based systems is suboptimal due to the lack of control over the time domain signals, large bandwidths and large PAPR leading to significant distortions and performance penalty. In addition, signal designed using inverse NFT and conventional formats are also very sensitive to linear and nonlinear responses of optical transmitter. As a results, advanced modulation formats, DSP and calibration techniques will be important research topic and challenges for future NFT research. ii) The impact of the deviations from the pure NLSE model (higher-order dispersion, polarization effects, Raman effect) on the properties and quality of NFT-based methods has yet to be carefully assessed. iii) The SE of NFT-based methods and of the nonlinear optical fibres has to be improved and elucidated further to overcome the SE limitation of "traditional" systems. This item also implies the optimization of the NFD modulation and invention of the new specific high SE formats for the NFT-based transmission. iv) Polarization division multiplexing with the use of NFT and Manakov equation integrability has yet to be developed. v) The numerical computation of the forward and inverse NFT becomes more and more difficult as the energy of the signal increases. With the NFT being a nonlinear operation, eventually all numerical algorithms break down [143]. While 
we expect that these issues can be fixed by improving the algorithms, e.g., by employing higher precision arithmetic of some form, it is also clear that this will increase the numerical costs. The development of NFT algorithms for the high energy regime and study of their complexity is an important open question.

\section{FUNDING INFORMATION}

This work was supported by the UK EPSRC Programme Grant UNLOC EP/J017582/1, ERC project ULTRALASER and Grant of the Ministry of Education and Science of the Russian Federation (agreement No. 14.B25.31.0003).

\section{SUPPLEMENTARY DOCUMENTS}

See Supplementary Material for supporting content.

\section{REFERENCES}

1. Agrawal, G.P. Fiber-Optic Communication Systems, 4th ed. (Wiley-Blackwell, 2010).

2. M. Cvijetic and I. B. Djordjevic, Advanced Optical Communication Systems and Networks (Artech House, 2013).

3. P. J. Winzer, "Spatial Multiplexing in Fiber Optics: The 10X Scaling of Metro/Core Capacities," Bell Labs Tech. J. 19, 2230 (2014).

4. P. J. Winzer, "Scaling Optical Fiber Networks: Challenges and Solutions," Optics \& Photonics News, 26 28-35 (2015).

5. R. J. Essiambre, R. W. Tkach, and R. Ryf, "Fiber nonlinearity and capacity: Single mode and multimode fibers," in Optical Fiber Telecommunications, Vol. VIB: Systems and Networks, 6th ed., Chpt. 1, edts. I. Kaminow, T. Li, and A.E. Willner, pp. 1-43 (Academic Press, 2013).

6. P. P. Mitra and J. B. Stark, "Nonlinear limits to the information capacity of optical fiber communications," Nature 411, 1027-1030 (2001).

7. R.-J. Essiambre, G. Kramer, P. J. Winzer, G. J. Foschini, and B. Goebel, "Capacity limits of optical fiber networks," J. Lightwave Technol. 28, 662-701 (2010).

8. A. D. Ellis, J. Zhao, and D. Cotter, "Approaching the nonlinear Shannon limit," J. Lightwave Technol. 28, 423-433 (2010).

9. D. J. Richardson, "Filling the light pipe," Science 330, 327328 (2010).

10. D. J. Richardson, “New optical fibres for high-capacity optical communications," Phil. Trans. R. Soc. A 374, 20140441 (2016).

11. R. I. Killey and C. Behrens, "Shannon's theory in nonlinear systems," J. Mod. Optics 58, 1-10 (2011).

12. R.-J. Essiambre, G. J. Foschini, G. Kramer, and P. J. Winzer, "Capacity limits of infomration transport in fiber-optic networks," Phys. Rev. Lett. 101, 163901 (2008).

13. C. E. Shannon, "A mathematical theory of communication," Bell Syst. Tech. J. 27, 379-423 (1948).

14. J. G. Proakis, Digital Communications (McGraw-Hill, 2001).

15. P. Bayvel, R. Maher, T. Xu, G. Liga, N. A. Shevchenko, D. Lavery, A. Alvarado, and R. I. Killey, "Maximizing the optical network capacity," Phil. Trans. R. Soc. A 374, 20140440 (2016).

16. L. B. Du, D. Rafique, A. Napoli, B. Spinnler, A. D. Ellis, M. Kuschnerov, and A. J. Lawery, "Digital Fiber Nonlinearity Compensation: Toward 1-Tb/s transport," IEEE Signal Processing Mag. 31, 46-56 (2014).
17. D. J. Richardson, J. M. Fini, and L. E. Nelson, "Spacedivision multiplexing in optical fibres," Nat. Photonics 7, 354-362 (2013).

18. E. Ip and J. Kahn. "Compensation of dispersion and nonlinear impairments using digital backpropagation," J. Lightwave Technol. 26, 3416-3425 (2008).

19. C. Xi, L. Xiang, S. Chandrasekhar, B. Zhu, and R. W. Tkach, "Experimental demonstration of fiber nonlinearity mitigation using digital phase conjugation," in Technical Digest of Optical Fiber Communication Conference and Exposition (OFC/NFOEC) and the National Fiber Optic Engineers Conference, paper OTh3C.1, 2012.

20. S. L. Jansen, D. Van den Borne, B. Spinnler, S. Calabro, H. Suche, P. M. Krummrich, G.-D. Khoe, and H. de Waardt, "Optical phase conjugation for ultra long-haul phase-shiftkeyed transmission," J. Lightwave Technol., 24, 54-64 (2006).

21. I. D. Phillips, M. Tan , M. F. C. Stephens, M. E. McCarthy, E. Giacoumidis, S. Sygletos, P. Rosa, S. Fabbri, S. T. Le, T. Kanesan, S. K. Turitsyn, N. J. Doran, P. Harper, and A. D. Ellis, "Exceeding the Nonlinear-Shannon Limit using Raman Laser Based Amplification and Optical Phase Conjugation," in Optical Fiber Communication Conference (OFC), Los Angeles, USA, paper M3C.1, 2014.

22. A. R. C. X. Liu, P. J. Winzer, R. W. Tkach, and S. Chandrasekhar, "Phase-conjugated twin waves for communication beyond the Kerr nonlinearity limit," Nat. Photonics 7, 560-568 (2013).

23. D. Rafique, "Fiber Nonlinearity Compensation: Commercial Applications and Complexity Analysis," J. Lightwave Technol. 34, 544-553 (2016).

24. J. E. Prilepsky and S. K. Turitsyn, "Eigenvalue communications in nonlinear fiber channels." In Odyssey of Light in Nonlinear Optical Fibers: Theory and Applications, Chpt. 18, edts. K. Porsezian and R. Ganapathy, pp. 459-490 (CRC Press, 2015).

25. P. K. A. Wai, C. R. Menyuk, Y. C. Lee, and H. H. Chen, "Nonlinear pulse propagation in the neighborhood of the zero-dispersion wavelength of monomode optical fibers," Opt. Lett. 11, 464-466 (1986).

26. L. F. Mollenauer and J. P. Gordon, Solitons in Optical Fibers: Fundamentals and Applications (Academic Press, 2006).

27. A. Hasegawa and Y. Kodama, Solitons in Optical Communications (Oxford University Press, 1995).

28. C. R. Menyuk, "Pulse propagation in an elliptically birefringent Kerr medium," IEEE J. Quant. Electron. 25, 26742682 (1989).

29. L.F. Mollenauer, K. Smith, J.P. Gordon, and C.R. Menyuk, "Resistance of solitons to the effects of polarization dispersion in optical fibers," Opt. Lett. 14, 1219-1221 (1989).

30. J. P. Gordon and H. Kogelnik, "PMD fundamentals: Polarization mode dispersion in optical fibers," Proc. Natl. Acad. Sci. USA 97, 4541-4550 (2000).

31. J. P. Gordon, "Theory of the soliton self-frequency shift," Opt. Lett. 11, 662-664 (1986).

32. E. M. Dianov, A. V. Luchnikov, A. N. Pilipetskii, and A. N. Starodumov, "Electrostriction mechanism of soliton interaction in optical fibers," Opt. Lett. 15, 314-316 (1990).

33. Y. S. Kivshar and G. P. Agrawal, Optical Solitons: From Fibers to Photonic Crystals (Academic Press, 2003).

34. E. Iannoe, F. Matera, A. Mecozzi, and M. Settembre, Nonlinear Optical Communication Networks (John Wiley \& Sons, New York, 1998). 
35. M. J. Ablowitz and H. Segur, Solitons and the Inverse Scattering Transform (SIAM, 1981).

36. G. L. Lamb, Jr. Elements of soliton theory (Wiley, 1980).

37. J. D. Ania-Castañón, “Quasi-lossless transmission using second-order Raman amplification and fibre Bragg gratings," Opt. Express 12, 4372-4377 (2004).

38. J. D. Ania-Castañón, T. J. Ellingham, R. Ibbotson, X. Chen, L. Zhang, and S. K. Turitsyn, "Ultralong Raman fibre lasers as virtually lossless optical media," Phys. Rev. Lett. 96, 023902 (2006).

39. J. D. Ania-Castañón, V. Karalekas, P. Harper, and S. K. Turitsyn, "Simultaneous Spatial and Spectral Transparency in Ultralong Fiber Lasers," Phys. Rev. Lett. 101, 123903 (2008).

40. V. E. Zakharov and A. B. Shabat, "Exact theory of 2-dimensional self-focusing and one-dimensional selfmodulation of waves in nonlinear media," Sov. Phys.-JETP 34, 62-69 (1972).

41. N. J. Ablowitz, D. J. Kaup, A. C. Newell, and H. Segur, H. "The inverse scattering transform-Fourier analysis for nonlinear problems," Stud. Appl. Math. 53, 249-315 (1974).

42. A. Hasegawa and T. Nyu, "Eigenvalue communication," J. Lightwave Technol. 11, 395-399 (1993).

43. J. Yang, Nonlinear Waves in Integrable and Nonintegrable Systems (SIAM, 2010).

44. M. I. Yousefi and F. R. Kschischang, "Information transmission using the nonlinear Fourier transform, Part I: Mathematical Tools." IEEE Trans. Inform. Theory 60, 4312-4328 (2014).

45. M. I. Yousefi and F. R. Kschischang, "Information transmission using the nonlinear Fourier transform, Part II: Numerical Mathods." IEEE Trans. Inform. Theory 60, 4329-4345 (2014).

46. M. I. Yousefi and F. R. Kschischang, "Information transmission using the nonlinear Fourier transform, Part III: Spectrum Modulation." IEEE Trans. Inform. Theory 60, 43464369 (2014).

47. S. V. Manakov, "On the theory of two-dimensional stationary self focussing of electromagnetic waves." Sov. Phys.JETP 38, 248-253 (1974).

48. S. Oda, A. Maruta, and K. Kitayama, "All-optical quantization scheme based on fiber nonlinearity." IEEE Photon. Techn. Lett. 16, 587-589 (2004).

49. J. E. Prilepsky, S. A. Derevyanko, and S. K. Turitsyn, "Nonlinear spectral management: linearization of the lossless fiber channel." Opt. Express 21, 24344-24367 (2013).

50. E. G. Turitsyna and S. K. Turitsyn, “Digital signal processing based on inverse scattering transform." Opt. Lett. 38, 4186-4188 (2013).

51. J. E. Prilepsky, S. A. Derevyanko, K. J. Blow, I. Gabitov, and S. K. Turitsyn, "Nonlinear Inverse Synthesis and Eigenvalue Division Multiplexing in Optical Fiber Channels." Phys. Rev. Lett. 113, 013901 (2014).

52. S. T. Le, J. E. Prilepsky, and S. K. Turitsyn, "Nonlinear inverse synthesis for high spectral efficiency transmission in optical fibers." Opt. Express 22, 26720-26741 (2014).

53. S. T. Le, J. E. Prilepsky, and S. K. Turitsyn, "Nonlinear inverse synthesis technique for optical links with lumped amplification." Opt. Express 23, 8317-8328 (2015).

54. S. T. Le, J. E. Prilepsky, M. Kamalian, P. Rosa, M. Tan, J. D. Ania-Castanon, P. Harper, and S. K. Turitsyn, "Modified Nonlinear Inverse Synthesis for Optical Links with Distributed Raman Amplification," in 41st European Conference on Optical Communications (ECOC), Valencia, Spain, paper Tu 1.1.3, 2015.

55. S. T. Le, J. E. Prilepsky, P. Rosa, J. D. Ania-Castanon, and S. K. Turitsyn, "Nonlinear Inverse Synthesis for Optical Links with Distributed Raman Amplification", J. Lightwave Technol. 34, 1778-1786 (2016).

56. S. T. Le, S. Wahls, D. Lavery, J. E. Prilepsky, and S. K. Turitsyn, "Reduced Complexity Nonlinear Inverse Synthesis for Nonlinearity Compensation in Optical Fiber Links", in Proceedings of Conference on Lasers and Electro-Optics/Europe and the European Quantum Electronics Conference(CLEO/EuropeEQEC), Munich, Germany, paper CI_3_2, 2015.

57. S. T. Le, I. D. Philips, J. E. Prilepsky, M. Kamalian, A. D. Ellis, P. Harper and S. K. Turitsyn, "Achievable Information Rate of Nonlinear Inverse Synthesis Based 16QAM OFDM Transmission"in 42st European Conference on Optical Communications (ECOC), Germany, paper Th.2.PS2.SC5, 2016

58. I. Tavakkolnia and M. Safari, "Signalling over nonlinear fibre-optic channels by utilizing both solitonic and radiative spectra," in IEEE European Conference on Networks and Communications (EuCNC), Paris, France, pp. 103-107, 2015.

59. A. Aref, S. T. Le and H. Buelow, "Demonstration of fully nonlinear spectrum modulated system in the highly nonlinear optical transmission regime" in European Conference on Optical Communication (ECOC), Germany, paper Th.3.B.2, 2016.

60. S. Hari, F. Kschischang, and M. Yousefi, "Multi-eigenvalue communication via the nonlinear Fourier transform," in 27th Biennial Symposium on Communications (QBSC), Kingston, ON, Canada, pp. 92-95, 2014.

61. Z. Dong, S. Hari, T. Gui, K. Zhong, M. I. Yousefi, C. Lu, P.K. A. Wai, F. R. Kschischang, and A. P. T. Lau, "Nonlinear Frequency Division Multiplexed Transmissions based on NFT," IEEE Photon. Tech. Lett. 27, 1621-1623 (2015).

62. H. Bülow, "Experimental assessment of nonlinear Fourier transformation based detection under fiber nonlinearity." In European Conference on Optical Communications (ECOC 2014), Cannes, France, paper We.2.3.2, 2014.

63. H. Bülow, "Experimental Demonstration of Optical Signal Detection Using Nonlinear Fourier Transform," J. Lightwave Technol. 33, 1433-1439 (2015).

64. H. Bülow, "Nonlinear Fourier Transformation Based Coherent Detection Scheme for Discrete Spectrum." In Optical Fiber Communication Conference (OFC), Los Angeles, USA, paper W3K.2, 2015.

65. V. Aref, H. Bülow, K. Schuh, and W. Idler, "Experimental Demonstration of Nonlinear Frequency Division Multiplexed Transmission," in 41st European Conference on Optical Communications (ECOC), Valencia, Spain, paper Tu 1.1.2, 2015. arXiv:1508.02577.

66. H. Terauchi and A. Maruta, "Eigenvalue Modulated Optical Transmission System Based on Digital Coherent Technology," in The 10th Conference on Lasers and Electro-Optics Pacific Rim, and the 18th OptoElectronics and Communications Conference/Photonics in Switching (CLEO-PR \& OECC/PS), Kyoto, Japan, Paper WR2-5, 2013.

67. H. Terauchi, Y. Matsuda, A. Toyota, and A. Maruta, “Noise Tolerance of Eigenvalue Modulated Optical Transmission System Based on Digital Coherent Technology," in The 19th OptoElectronics and Communications Conference/Australian Conference on Optical Fibre Technology (OECC/ACOF), Melbourne, Australia, Paper 542, 2014.

68. Y. Matsuda, H. Terauchi, and A. Maruta, "Design of Eigenvalue-multiplexed Multi-level Modulation Optical 
Transmission System," in The 19th OptoElectronics and Communications Conference/Australian Conference on Optical Fibre Technology (OECC/ACOF), Melbourne, Australia, Paper TH12B3, 2014

69. A. Maruta, “Eigenvalue Modulated Optical Transmission System (invited)," in The 20th OptoElectronics and Communications Conference (OECC), Shanghai, China, Paper JThA.21, 2015

70. A. Toyota and A. Maruta, "Wavelength division multiplexed optical eigenvalue modulated system," in Tyrrhenian International Workshop on Digital Comminications (TIWDC), Florence, Italy, pp. 43-45, 2015.

71. A. Maruta, A. Toyota, Y. Matsuda, and Y. Ikeda, “Experimental demonstration of long haul transmission of eigenvalue modulated signals," Tyrrhenian International Workshop on Digital Comminications (TIWDC), Florence, Italy, pp. 28-30, 2015.

72. A. Maruta and Y. Matsuda, "Polarization Division Multiplexed Optical Eigenvalue Modulation," in International Conference on Photonics in Switching (PS), Florence, Italy, pp. 265-267, 2015.

73. S. Wahls, S. T. Le, J. E. Prilepsky, H. V. Poor, and S. K. Turitsyn, "Digital Backpropagation in the Nonlinear Fourier Domain," in Proceedings of IEEE 16th International Workshop in Signal Processing Advances in Wireless Communications (SPAWC), Stockholm, Sweden, pp. 445-449, 2015.

74. Q. Zhang and T. H. Chan, "A Gaussian Noise Model of Spectral Amplitudes in Soliton Communication Systems", in Proceedings of IEEE 16th International Workshop in Signal Processing Advances in Wireless Communications (SPAWC), Stockholm, Sweden, pp. 455-459, 2015.

75. Q. Zhang and T. H. Chan, "A spectral domain noise model for optical fibre channels," in IEEE International Symposium on Information Theory (ISIT), Hong Kong, China, paper WeAM2-9.1,2015.

76. E. Meron, M. Feder, and M. Shtaif, "On the achievable communication rates of generalized soliton transmission systems," arXiv:1207.0297 (2012).

77. N. A. Shevchenko, J. E. Prilepsky, S. A. Derevyanko, A. Alvarado, P. Bavel, and S. K. Turitsyn, "A Lower Bound on the per Soliton Capacity of the Nonlinear Optical Fibre Channel," in IEEE Information Theory Workshop (ITW), Jeju Island, Korea, pp. 104-108, 2015.

78. M. Tan, P. Rosa, I. D. Phillips, and P. Harper, "Long-haul Transmission Performance Evaluation of Ultra-long Raman Fiber Laser Based Amplification Influenced by Second Order Co-pumping," in Asia Communications and Photonics Conference, OSA Technical Digest, paper ATh1E.4, 2014.

79. L. Poladian, "Iterative and noniterative design algorithms for Bragg gratings," Opt. Fiber Technol. 5, 215-222 (1999).

80. J. Skaar, L. Wang, and T. Erdogan, "On the synthesis of fiber Bragg gratings by layer peeling." IEEE J. Quant. Electr. 37, 165-173 (2001).

81. A. Rosenthal and M. Horowitz, "Inverse scattering algorithm for reconstructing strongly reflecting fiber Bragg gratings." IEEE J. Quant. Electr. 39, 1018-1026 (2003).

82. C. F. Elliott, Handbook of digital signal processing: engineering applications (Academic press, 2013).

83. S. Wahls and H. V. Poor, "Fast Numerical Nonlinear Fourier Transforms," IEEE Trans. Inform. Theory 61, 69576974 (2015).

84. M. Kamalian Kopae, J. E. Prilepsky, S. T. Le, and S. K. Turitsyn, "Optical communication based on the periodic nonlin- ear Fourier transform signal processing," IEEE 6th International Conference on Photonics (ICP), Kuching, Malaysia, pp. 1-3, 2016.

85. M. Kamalian Kopae, J. E. Prilepsky, S. T. Le, and S. Turitsyn, "Periodic nonlinear Fourier transform based optical communication systems in a band-limited regime," in Advanced Photonics (IPR, NOMA, Sensors, Networks, SPPCom, SOF). OSA Technical Digest, Vancouver, Canada, paper JTu4A.34, 2016.

86. M. Kamalian, J. E. Prilepsky, S. T. Le, and S. K. Turitsyn, "Periodic nonlinear Fourier transform for fiber-optic communications, Part I: theory and numerical methods," Opt. Express 24, 18353-18369 (2016).

87. M. Kamalian, J. E. Prilepsky, S. T. Le, and S. K. Turitsyn, "Periodic nonlinear Fourier transform for fiber-optic communications, Part II: eigenvalue communication," Opt. Express 24, 18370-18381 (2016).

88. M. Kamalian, J. E. Prilepsky, S. T. Le, and S. K. Turitsyn, "Periodic Nonlinear Fourier Transform Based Transmissions with High Order QAM Formats," in 42st European Conference on Optical Communications (ECOC), Germany, pp. 1-3, 2016.

89. O. R. Its and V. P. Kotlyarov, "Explicit formulas for the solutions of a nonlinear Schrodinger equation," Doklady Akad. Nauk Ukrainian SSR, ser. A, vol. 10, 965-968 (1976); English translation available online at http://arxiv.org/abs/1401.4445v1.

90. Y. Ma and M. J. Ablowitz, "The Periodic Cubic Schrödinger Equation," Stud. Appl. Math. 65, 113-158 (1981).

91. E. R. Tracy and H. H. Chen, "Nonlinear self-modulation: An exactly solvable model," Phys. Rev. A 37, 815-839 (1988).

92. A. Osborne, Nonlinear Ocean Waves and the Inverse Scattering Transform, 1st ed. (Academic Press, 2010).

93. A. Bobenko and C. Klein, eds. Computational approach to Riemann surfaces, No. 2013, (Springer Science and Business Media, 2011).

94. B. Deconinck, M. S. Patterson, and C. Swierczewski, "Computing the Riemann Constant Vector," preprint, available online at https://depts.washington.edu/bdecon/papers/pdfs/rcv.pdf (2015).

95. J. Frauendiener and C. Klein, "Computational approach to hyperelliptic Riemann surfaces," Lett. Math. Phys. 105, 379-400 (2015).

96. B. Deconinck, H. Heil, A. Bobenko, M. Van Hoeij, and M. Schmies, "Computing Riemann theta functions," Math. Comput. 73, 1417-1442 (2004).

97. C. Swierczewski and B. Deconinck, "Computing Riemann theta functions in Sage with applications," Math. Comput. Simulat., Available online http:/ /dx.doi.org/10.1016/j.matcom.2013.04.018 (2013).

98. S. Wahls and H. V. Poor, "Introducing the fast nonlinear Fourier transform," in Proceedings of International Conference on Acoustics, Speech, and Signal Processing (ICASSP), Vancouver, Canada, pp. 5780-5784, 2013.

99. S. Hari and F. R. Kschischang, "Bi-Directional Algorithm for Computing Discrete Spectral Amplitudes in the NFT," J. Lightwave Technol. 34, 3529-3537 (2016).

100. L. Fermo, C. van der Mee, and S. Seatzu, "Numerical solution of the direct scattering problem for the nonlinear Schrödinger equation," in Tyrrhenian International Workshop on Digital Comminications (TIWDC), Florence, Italy, pp. 5-8, 
2015.

101. G. Boffetta and A. Osborne, "Computation of the direct scattering transform for the nonlinear schroedinger equation," J. Comput. Phys. 102, 252-264 (1992).

102. S. Burtsev, R. Camassa, and I. Timofeyev, “Numerical algorithms for the direct spectral transform with applications to nonlinear Schrödinger type systems," J. Comput. Phys. 147, 166-186 (1998).

103. J. E. Prilepsky, S.A. Derevyanko, and S. K. Turitsyn, “Conversion of a chirped Gaussian pulse to a soliton or a bound multisoliton state in quasi-lossless and lossy optical fiber spans," J. Opt. Soc. Am. B 24, 1254-1261 (2007).

104. J. E. Prilepsky and S. A. Derevyanko, "Breakup of a multisoliton state of the linearly damped nonlinear Schrödinger equation," Phys. Rev. E 75, 036616 (2007).

105. M. J. Ablowitz and J. F. Ladik, "Nonlinear differentialdifference equations," J. Math. Phys. 16, 598-603 (1975).

106. M. J. Ablowitz and J. F. Ladik, "A difference scheme and inverse scattering," Stud. Appl. Math. 55, 213-229 (1976).

107. J. K. Brenne and J. Skaar, "Design of grating-assisted codirectional couplers with discrete-scattering algorithms," J. Lightwave Technol. 21, 254-263 (2003).

108. S. Wahls and H. V. Poor, "Inverse Nonlinear Fourier Transforms Via Interpolation: The Ablowitz-Ladik Case," in Proceeding of International Symposium on Mathematical Theory of Networks and Systems (MTNS), Groningen, The Netherlands, pp. 1848-1855, 2014.

109. A. R. Osborne, “The hyperelliptic inverse scattering transform for the periodic, defocusing nonlinear Schroedinger equation," J. Comput. Phys. 109, 93-107 (1993).

110. L. L. Frumin, O. V. Belai, E. V. Podivilov, and D. A. Shapiro, "Efficient numerical method for solving the direct Zakharov-Shabat scattering problem," J. Opt. Soc. Am. B 32, 290-295 (2015).

111. G. H. Song and S. Y. Shin, "Design of corrugated waveguide filters by the Gel'fand-Levitan-Marchenko inverse scattering method," J. Opt. Soc. Am. A 2, 1905-1915 (1985).

112. F. Ahmad and M. Razzagh, "A numerical solution to the Gel'fand-Levitan-Marchenko equation." Appl. Math. Comput. 89, 31-39 (1998).

113. R. Feced, M. N. Zervas, and M. A. Muriel, “An efficient inverse scattering algorithm for the design of nonuniform Bragg gratings," IEEE J. Quant. Electr. 35, 1105-1115 (1999).

114. J. Skaar and R. Feced, "Reconstruction of gratings from noisy reflection data," J. Opt. Soc. Am. A 19, 2229-2237 (2002).

115. J. Skaar and O. H. Waagaard, "Design and characterization of finite-length fiber gratings. Design and characterization of finite-length fiber gratings," IEEE J. Quant. Electr. 39, 1238-1245 (2003).

116. S. K. Turitsyn and S. A. Derevyanko, "Soliton-based discriminator of noncoherent optical pulses," Phys. Rev. A 78, 063819 (2008).

117. S. A. Derevyanko and J. E. Prilepsky, "Random input problem for the nonlinear Schrödinger equation," Phys. Rev. E 78, 046610 (2008).

118. S. A. Derevyanko, "Design of a flat-top fiber Bragg filter via quasi-random modulation of the refractive index," Opt. Lett. 33, 2404-2406 (2008).

119. S. A. Derevyanko, "Appearance of bound states in random potentials with applications to soliton theory," Phys. Rev. E 84, 016601 (2011).

120. S. Wahls and H. V. Poor, "Fast inverse nonlinear Fourier transform for generating multisolitons in optical fiber," in Proeedings of IEEE International Symposium on Information Theory (ISIT), Hong Kong, China, pp. 1676-1680, 2015.

121. P. Frangos and D. Jaggard, "A numerical solution to the Zakharov-Shabat inverse scattering problem," IEEE Trans. Antennas Propag. 39, 74-79 (1991).

122. G. B. Xiao and K. Yashiro, "An efficient algorithm for solving Zakharov-Shabat inverse scattering problem," IEEE Trans. Antennas Propag. 50, 807-811 (2002).

123. A. Buryak, J. Bland-Hawthorn, and V. Steblina, "Comparison of inverse scattering algorithms for designing ultrabroadband fiber Bragg gratings," Opt. Express 17, 19952004 (2009).

124. C. Papachristos and P. Frangos, "Design of corrugated optical waveguide filters through a direct numerical solution of the coupled Gel'fand-Levitan-Marchenko integral equations," J. Opt. Soc. Am. A 19, 1005-1012 (2002).

125. O. V. Belai, L. L. Frumin, E. V. Podivilov, O. Y. Schwarz, and D. A. Shapiro, "Finite Bragg grating synthesis by numerical solution of Hermitian Gel'fand-LevitanMarchenko equations," J. Opt. Soc. Am. B 23, 2040-2045 (2006).

126. O. V. Belai, L. L. Frumin, E. V. Podivilov, and D. A. Shapiro, "Efficient numerical method of the fiber Bragg grating synthesis," J. Opt. Soc. Am. B 24, 1451-1457 (2007).

127. W. F. Trench, "An algorithm for the inversion of finite toeplitz matrices," J. Soc. Indust. Appl. Math. 12, 515-522 (1964).

128. S. Zohar, "Toeplitz Matrix Inversion: The Algorithm of W. F. Trench," Journal of the ACM 16, 592-561 (1969).

129. S. Civelli, L. Barletti, and M. Secondini, "Numerical Methods for the Inverse Nonlinear Fourier Transform," in Tyrrhenian International Workshop on Digital Comminications (TIWDC), Florence, Italy, pp. 13-16, 2015.

130. A. Arico, G. Rodriguez, and S. Seatzu, "Numerical solution of the nonlinear Schrödinger equation, starting from the scattering data," Calcolo 48, 75-88 (2011).

131. T. Trogdon and S. Olver, "Numerical inverse scattering for the focusing and defocusing nonlinear Schrödinger equations," Proc. R. Soc. A 469, 20120330 (2013).

132. L. Rabiner, R. Schafer, and C. Rader, "The chirp ztransform algorithm," IEEE Trans. Audio Electroacoust. 17, 86-92 (1969).

133. P. Boito, Y. Eidelman, L. Gemignami, and I. Gohberg, "Implicit QR with compression," Indag. Math. 23, 733-761 (2012).

134. J. L. Aurentz, T. Mach, R. Vandebril, and D. S. Watkins, "Fast and Backward Stable Computation of Roots of Polynomials," SIAM J. Matrix Anal. Appl. 36, 942-973 (2015).

135. V. Vaibhav and S. Wahls, "Multipoint Newton-type Nonlinear Fourier Transform for Detecting Multi-Solitons," Proc. Optical Fiber Communication Conference (OFC), Anaheim, USA, paper W2A.34, 2016.

136. S. Wahls and V. Vaibhav, "Fast generation of multisolitons using the Darboux transform," in Munich Wksp. Inf. Theory Optical Fiber (MIO), Tech. Univ. Munich, Dec. 2015. Slides: http:/ / bit.ly/2dD61Bh.

137. S. Wahls and V. Vaibhav, "Fast Inverse Nonlinear Fourier Transforms for Fiber Bragg Grating Design and Related Problems ," Preprint, Jun. 2016, arXiv:1607.01305 [cs.IT].

138. S. Wahls and V. Vaibhav, "Introducing the Fast Inverse NFT," Submitted to OFC 2017.

139. W. K. McClary, "Fast seismic inversion," Geophys. 48, 
1371-1372 (1983).

140. S. Hari, M. I. Yousefi, and F. R. Kschischang, "Multieigenvalue Communication", J. Lightwave Technol. 34, 31103117 (2016).

141. A. Span and S. ten Brink, "Optical Communications Using the Nonlinear Fourier Transform," in Munich Wksp. Inf. Theory Optical Fiber (MIO), Tech. Univ. Munich, Dec. 2015. Slides: http:/ / bit.ly/2ePRyCZ

142. H. Buelow, V. Aref and W. Idler, "Transmission of Waveform Determined by 7 Eigenvalues with PSK-Modulated Spectral Amplitude"in 42st European Conference on Optical Communications (ECOC), Germany, paper Tu.3.E.2, 2016

143. I. T. Lima, V. S. Grigoryan, M. O'Sullivan, and C. R. Menyuk, "Computational complexity of nonlinear transforms applied to optical communications systems with normal dispersion fibers," Proc. IEEE Photonics Conference (IPC), Reston, USA, pp. 277-278, 2015.

144. S. T. Le, I. D. Philips, J. E. Prilepsky, P. Harper, A. D. Ellis, and S. K. Turitsyn, "Demonstration of Nonlinear Inverse Synthesis Transmission over Transoceanic Distances," J. Lightwave Technol. 34, 2459-2466 (2016).

145. S. T. Le, I. Philips, J. Prilepsky, P. Harper, N. Doran, A. D. Ellis, and S. Turitsyn, "First Experimental Demonstration of Nonlinear Inverse Synthesis Transmission over Transoceanic Distances," in Optical Fiber Communication Conference (OFC), OSA Technical Digest, Anaheim, USA, paper Tu2A.1, 2016.

146. E. Agrell, A. Alvarado, and F. R. Kschishang "Implications of Information Theory in Optical Fibre Communications,", Phil. Trans. R. Soc. A, 374, 20140438 (2016).

147. E. Forestieri and M. Secondini, "The Nonlinear FiberOptic Channel: Modeling and Achievable Information Rate," in Progress In Electromagnetics Research Symposium (PIERS), Prague, Czech Republic, pp. 1276-1283, 2015.

148. J. P. Gordon and H. A. Haus, "Random walk of coherently amplified solitons in optical fiber transmission," Opt. Lett. 11, 665-667 (1986).

149. Q. Zhang and T. H. Chan, "Achievable rates of soliton communication systems," in IEEE International Symposium on Information Theory (ISIT), Barcelona, Spain, pp. 605-609 (2016).

150. S. A. Derevyanko, S. K. Turitsyn, and D. A. Yakushev, "Non-Gaussian Statistics of an Optical Soliton in the Presence of Amplified Spontaneous Emission," Opt. Lett., 28, 2097-2099 (2003).

151. S. A. Derevyanko, S. K. Turitsyn, and D. A. Yakushev, "Fokker-Planck Equation Approach to the Description of Soliton Statistics in Optical Fiber Transmission Systems", J. Opt. Soc. Am. B, 22, 743-752 (2005).

152. P. Kazakopoulos and A. L. Moustakas, "On the soliton spectral efficiency in non-linear optical fibers," in IEEE International Symposium on Information Theory (ISIT), Barcelona, Spain, pp. 610-614 (2016).

153. N. A. Shevchenko, S. A. Derevyanko, J. E. Prilepsky, A. Alvarado, P. Bavel, and S. K. Turitsyn, "A Lower Bound on the Capacity of the Noncentral Chi Channel with Applications to Soliton Amplitude Modulation,"submitted to IEEE Tranf. Inf. Theory (Sep. 2016); arXiv:1609.02318 (2016).

154. S. A. Derevyanko, J. E. Prilepsky, and D. A. Yakushev, "Statistics of a noise-driven Manakov soliton," J. Phys. A: Math. Gen. 39, 1297-1309 (2006).

155. S. A. Derevyanko, J. E. Prilepsky, and S. K. Turitsyn, “Capacity estimates for optical transmission based on the non- linear Fourier transform," Nat. Commun. 7, 12710 (2016).

156. D. J. Kaup, "Perturbation expansion for Zakharov-Shabat inverse scattering transform," SIAM J. Appl. Math. 31, 121133 (1976).

157. D. J. Kaup and A. C. Newell, "Solitons as particles, oscillators, and in slowly changing media - singular perturbationtheory," Proc. Roy. Soc. Lond. A Mat. 361, 413-446 (1978).

158. M. S. Pinsker, Information and Informational Stability of Random Variables and Processes, pp. 160-201 (Holden Day, 1964).

159. M. I. Yousefi and X. Yangzhang, "Linear and Nonlinear Frequency-Division Multiplexing", arXiv:1207.0297 (2016).

160. S. T. Le, I. D. Philips, J. E. Prilepsky, M. Kamalian, A. D. Ellis, P. Harper and S. K. Turitsyn, “Equalization-Enhanced Phase Noise in Nonlinear Inverse Synthesis Transmissions"in 42st European Conference on Optical Communications (ECOC), Germany, paper Tu.3.B.2, 2016. 